ALGEBRAIC METHODS IN LOGIC AND IN COMPUTER SCIENCE

BANACH CENTER PUBLICATIONS, VOLUME 28

INSTITUTE OF MATHEMATICS

POLISH ACADEMY OF SCIENCES

WARSZAWA 1993

\title{
COMPACTNESS AND \\ LÖWENHEIM-SKOLEM PROPERTIES IN CATEGORIES OF PRE-INSTITUTIONS
}

\author{
ANTONINO SALIBRA \\ University of Pisa, Dip. Informatica \\ Corso Italia 40, I-56125 Pisa, Italy \\ E-mail: SALIBRA@DI.UNIPI.IT \\ GIUSEPPE SCOLLO \\ University of Twente, Fac. Informatica \\ P. O. Box 217, NL-7500AE Enschede, The Netherlands \\ E-mail: SCOLLO@CS.UTWENTE.NL
}

\begin{abstract}
The abstract model-theoretic concepts of compactness and Löwenheim-Skolem properties are investigated in the "softer" framework of pre-institutions [18]. Two compactness results are presented in this paper: a more informative reformulation of the compactness theorem for pre-institution transformations, and a theorem on natural equivalences with an abstract form of the first-order pre-institution. These results rely on notions of compact transformation, which are introduced as arrow-oriented generalizations of the classical, object-oriented notions of compactness. Furthermore, a notion of cardinal pre-institution is introduced, and a LöwenheimSkolem preservation theorem for cardinal pre-institutions is presented.
\end{abstract}

1. Introduction. In [18] we introduced the notion of pre-institution as an abstract notion of logical system, together with a notion of pre-institution transformation, which enables the transfer of logical reasoning, model-theoretic results and computing tools from one pre-institution to another.

The original target of our investigation was the notion of institution, which was introduced in [9] as a vehicle for the application of abstract model theory

1991 Mathematics Subject Classification: 03C95, 03B10, 18B99, 03G99, 68Q65.

This work was produced while the second author was on temporary leave at LIENS, DMI, École Normale Supérieure, 45 Rue d’Ulm, F-75230 Paris Cedex 05, France.

The paper is in final form and no version of it will be published elsewhere. 
to computer science. Motivation for the choice of that target was the experience gained in [14], relating to the translation of a number of logics into equational type logic $[13,12]$. We detected a striking commonality over the different translations, concerning representation of models, translation of sentences, and structure of completeness proofs. The search for a more general framework, where that commonality could be factored out, was just as natural. For such an aim, the category of institutions seemed to offer the obvious framework to work with. Two facts, however, indicated that this choice was not entirely obvious.

In the first place, the way in which the expressiveness results in [14] were obtained led us to observe that pointwise translation of sentences and models is not always easy to work with. More generally, we need to translate presentations (i.e. sets of sentences) to presentations, and to associate a class of models in the target logic with each model of the source logic. The notion of institution morphism proposed in [9] thus deserves generalization.

In the second place, we observed that not every feature of the institution concept had some rôle to play in the trial applications of our interest. The softness of the satisfaction condition, in particular, does not seem to have been weighed on sufficiently accurate scales.

The putting together of the two facts that occurred to our observation motivated thus the introduction of the less restrictive, albeit structurally weaker notion of pre-institution, together with an easier-to-use notion of morphism, which we called pre-institution transformation.

Now, in this paper we investigate a few most relevant concepts of abstract model theory, viz. compactness and Löwenheim-Skolem properties, in the even "softer" pre-institutions framework. In particular, we study the inheritance of these properties along pre-institution transformations.

Concerning compactness, the result in [18] is refined by introducing a notion of compact transformation, whereby the classical concept of compactness of a logical system, once formulated for the soft framework of pre-institutions, is, so to say, "stretched" along the transformation arrow, relating validity in the target to finiteness in the source.

Moreover, we show the contravariant inheritance of a general form of the Löwenheim-Skolem properties by "suitable" pre-institution transformations. To this purpose, the notion of pre-institution with cardinal numbers, or cardinal preinstitution, is introduced, since Löwenheim-Skolem properties make reference to the cardinality of models (and of symbol sets as well, in the more general forms of those properties).

Both in the case of compactness and in that of Löwenheim-Skolem properties, inheritance is "contravariant" in the sense that, if $\mathcal{T}: \mathcal{I} \rightarrow \mathcal{I}^{\prime}$ is a "suitable" preinstitution transformation and $\mathcal{I}^{\prime}$ has the property under consideration, then $\mathcal{I}$ has that property as well. The pre-institution transformation is to be "suitable" in the sense that it may have to fulfil certain requirements - such as (full) adequacy, finitarity, etc. - depending on the property under consideration. Essentially, thus, 
we are in the presence of a generalization of the well-known "reduction scheme" from abstract model theory that is outlined in [5].

A central place in the analysis developed in this paper is given to a result on the equivalence with an abstract form of the first-order pre-institution, i.e. the pre-institution arising from first-order logic. This result generalizes a key lemma in the proof of the first Lindström theorem that is given in [6].

The organization of the rest of this paper is as follows. First, for the sake of self-containedness, we recall from [18] the needed definitions and facts, in Sections 2 and 3 respectively, but with new arguments and motivations in Section 2, taking the target of the present work into account. In Section 4, notions of compact transformation are introduced, and the compactness theorem from [18] (recalled in Section 3), is then refined to a more informative theorem, linking compactness of a transformation with compactness of its source and target pre-institutions. In Section 5, two new concepts are introduced, viz. that of pre-institution with abstract sentences, or abstract pre-institution, and that of expansion-adequate transformation. These concepts, together with the results on compact transformations, play an essential rôle in the statement and proof of a theorem on the equivalence with the abstract, first-order pre-institution - the main result presented in Section 6. The notion of cardinal pre-institution is then introduced in Section 7, leading to a general form of Löwenheim-Skolem preservation theorem for cardinal pre-institutions, as worked out in this section. Finally, connections with related work are discussed in Section 8, and Section 9 closes the paper with an outline of topics for further investigation.

2. Basic notions and notations. A preliminary word about foundations. In this paper we use the term "set" in a rather comprehensive meaning, that generally includes proper classes. Whenever a need arises to exclude proper classes, we talk of "small sets". SET is thus actually a "metacategory" $\left({ }^{1}\right)$, according to [10].

Definition 2.1. A pre-institution is a 4 -tuple $\mathcal{I}=(\operatorname{Sig}$, Sen, Mod, $\vDash)$, with:

(i) Sig a category, whose objects are called signatures,

(ii) Sen : Sig $\rightarrow$ SET a functor, sending each signature $\Sigma$ to the set $\operatorname{Sen}(\Sigma)$ of $\Sigma$-sentences, and each signature morphism $\tau: \Sigma \rightarrow \Sigma^{\prime}$ to the mapping $\operatorname{Sen}(\tau)$ : $\operatorname{Sen}(\Sigma) \rightarrow \operatorname{Sen}\left(\Sigma^{\prime}\right)$ that translates $\Sigma$-sentences to $\Sigma^{\prime}$-sentences,

(iii) Mod : $\operatorname{Sig}^{\text {op }} \rightarrow$ SET a functor, sending each signature $\Sigma$ to the set $\operatorname{Mod}(\Sigma)$ of $\Sigma$-models, and each signature morphism $\tau: \Sigma \rightarrow \Sigma^{\prime}$ to the $\tau$-reduction function $\operatorname{Mod}(\tau): \operatorname{Mod}\left(\Sigma^{\prime}\right) \rightarrow \operatorname{Mod}(\Sigma)$

(iv) $\vDash:|\operatorname{Sig}| \rightarrow\|\operatorname{REL}\|$ a function $\left({ }^{2}\right)$, yielding a binary relation $\vDash_{\Sigma} \subseteq \operatorname{Mod}(\Sigma) \times$ $\operatorname{Sen}(\Sigma)$ for each signature $\Sigma$, viz. the satisfaction relation between $\Sigma$-models and $\Sigma$-sentences.

$\left({ }^{1}\right)$ Resting at some floor of "palais Grothendieck".

$\left({ }^{2}\right)$ REL is the category of sets with binary relations as morphisms; $\|C\|$ is the set of morphisms of the category $C$. 
By a slight notational abuse, we will write $\Sigma \in \operatorname{Sig}$ instead of $\Sigma \in|\operatorname{Sig}|$, and $\tau: \Sigma \rightarrow \Sigma^{\prime} \in \operatorname{Sig}$ instead of $\tau: \Sigma \rightarrow \Sigma^{\prime} \in\|\operatorname{Sig}\|$.

Definition 2.2. Let $\mathcal{I}=(\operatorname{Sig}$, Sen, Mod, $\vDash)$ be a pre-institution, $\tau: \Sigma \rightarrow \Sigma^{\prime}$ a signature morphism in Sig, $\varphi$ a $\Sigma$-sentence and $M^{\prime}$ a $\Sigma^{\prime}$-model. With henceforth adoption of the abbreviations: $\tau \varphi$ for $\operatorname{Sen}(\tau)(\varphi)$, and $M^{\prime} \tau$ for $\operatorname{Mod}(\tau)\left(M^{\prime}\right)$, we say that

(i) reduction preserves satisfaction in $\mathcal{I}$, or that $\mathcal{I}$ has the rps property (or that $\mathcal{I}$ is rps, for short), iff $\mathcal{I}$ meets the following requirement:

(†) $\forall \tau: \Sigma \rightarrow \Sigma^{\prime} \in \operatorname{Sig}, \forall \varphi \in \operatorname{Sen}(\Sigma), \forall M^{\prime} \in \operatorname{Mod}\left(\Sigma^{\prime}\right)$ :

$$
M^{\prime} \vDash_{\Sigma^{\prime}} \tau \varphi \Rightarrow M^{\prime} \tau \vDash_{\Sigma} \varphi .
$$

(ii) expansion preserves satisfaction in $\mathcal{I}$, or that $\mathcal{I}$ has the eps property (or that $\mathcal{I}$ is eps, for short), iff $\mathcal{I}$ meets the following requirement:

( ) $\forall \tau: \Sigma \rightarrow \Sigma^{\prime} \in \operatorname{Sig}, \forall \varphi \in \operatorname{Sen}(\Sigma), \forall M^{\prime} \in \operatorname{Mod}\left(\Sigma^{\prime}\right):$

$$
M^{\prime} \tau \vDash_{\Sigma} \varphi \Rightarrow M^{\prime} \vDash_{\Sigma^{\prime}} \tau \varphi .
$$

(iii) $\mathcal{I}$ preserves satisfaction, or that $\mathcal{I}$ has the ps property (or that $\mathcal{I}$ is ps, for short), iff $\mathcal{I}$ is both rps and eps.

An institution [8] is thus a pre-institution that preserves satisfaction and where model sets and reduction have categorial structure, that is, an institution rather has a functor Mod : Sig ${ }^{\text {op }} \rightarrow$ CAT, sending each signature $\Sigma$ to the category $\operatorname{Mod}(\Sigma)$ of $\Sigma$-models, and each signature morphism $\tau: \Sigma \rightarrow \Sigma^{\prime}$ to the $\tau$-reduction functor $\operatorname{Mod}(\tau): \operatorname{Mod}\left(\Sigma^{\prime}\right) \rightarrow \operatorname{Mod}(\Sigma)$. It seems interesting to investigate which properties of institutions do actually depend on requirements $(\dagger)$ and/or $(\ddagger)$ of Definition 2.2, and/or on the categorial structure of model sets and reduction, and which do not, thus holding for pre-institutions as well.

According to the motivation proposed in [18], we are interested in general tools for lifting results from one pre-institution to another pre-institution. Pointwise translation of sentences and models is not always easy to use for this purpose. For example, to recover and possibly further extend the results obtained in [14], we need to translate presentations to presentations, and to associate a set of models (in the target pre-institution) with each model of the source pre-institution. A suitable notion of pre-institution morphism serves this purpose, for which a few preliminaries are needed.

We recall that the powerset functor $\wp:$ SET $\rightarrow$ SET sends every set to the collection of its subsets $\left(^{3}\right)$, and every function $f: S \rightarrow S^{\prime}$ to the function yielding the $f$-image of each subset of $S$. The functor $\wp_{+}$is analogously defined, except that the empty set is excluded from the collection $\wp_{+}(S)$, for all sets $S$

In every pre-institution $\mathcal{I}=(\operatorname{Sig}, \operatorname{Sen}$, Mod, $\models)$ we thus define Pre $=\wp \circ$ Sen : $\mathrm{Sig} \rightarrow$ SET as the corresponding functor that sends each signature $\Sigma$ to the

$\left({ }^{3}\right) \wp$ lives in the elevator of "palais Grothendieck", thus, lifting its argument up one floor. 
set $\operatorname{Pre}(\Sigma)$ of $\Sigma$-presentations, and each signature morphism $\tau: \Sigma \rightarrow \Sigma^{\prime}$ to the mapping $\operatorname{Pre}(\tau): \operatorname{Pre}(\Sigma) \rightarrow \operatorname{Pre}\left(\Sigma^{\prime}\right)$ that translates $\Sigma$-presentations to $\Sigma^{\prime}$ presentations. For convenience, we often write $\mathcal{I}=($ Sig, Pre, Mod, $\vDash)$ instead of the more customary notation introduced in Definition 2.1.

Definition 2.3. A pre-institution transformation $\mathcal{T}: \mathcal{I} \rightarrow \mathcal{I}^{\prime}$, where $\mathcal{I}=$ (Sig, Pre, Mod, $\vDash)$ and $\mathcal{I}^{\prime}=\left(\mathrm{Sig}^{\prime}, \mathrm{Pre}^{\prime}, \mathrm{Mod}^{\prime}, \models^{\prime}\right)$ are pre-institutions, is a 3 -tuple $\mathcal{T}=\left(\operatorname{Si}_{\mathcal{T}}, \operatorname{Pr}_{\mathcal{T}}, \operatorname{Mo}_{\mathcal{T}}\right)$, with:

(i) $\operatorname{Si}_{\mathcal{T}}: \operatorname{Sig} \rightarrow \operatorname{Sig}^{\prime}$ a functor - we shall henceforth write $\Sigma_{\mathcal{T}}$ for $\operatorname{Si}_{\mathcal{T}}(\Sigma)$, and $\tau_{\mathcal{T}}$ for $\operatorname{Si}_{\mathcal{T}}(\tau)$,

(ii) $\operatorname{Pr}_{\mathcal{T}}: \operatorname{Pre} \rightarrow \operatorname{Pre}^{\prime} \circ \operatorname{Si}_{\mathcal{T}}$ a natural transformation, i.e. for each $\Sigma \in \operatorname{Sig}$ a function $\operatorname{Pr}_{\mathcal{T}_{\Sigma}}: \operatorname{Pre}(\Sigma) \rightarrow \operatorname{Pre}^{\prime}\left(\Sigma_{\mathcal{T}}\right)$ sending $\Sigma$-presentations to $\Sigma_{\mathcal{T}}$-presentations, such that for every signature morphism $\tau: \Sigma_{1} \rightarrow \Sigma_{2}$ in Sig the following diagram commutes:

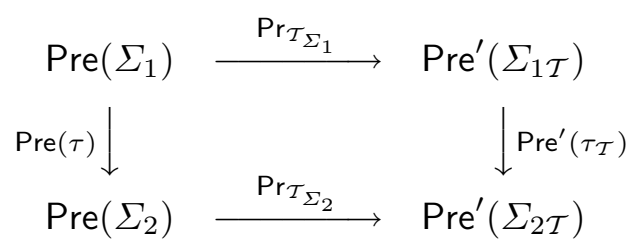

(iii) $\mathrm{Mo}_{\mathcal{T}}: \operatorname{Mod} \rightarrow \wp_{+} \circ \operatorname{Mod}^{\prime} \circ \mathrm{Si}_{\mathcal{T}}$ a natural transformation, i.e. for each $\Sigma \in$ Sig a map $\operatorname{Mo}_{\mathcal{T}_{\Sigma}}: \operatorname{Mod}(\Sigma) \rightarrow \wp_{+}\left(\operatorname{Mod}^{\prime}\left(\Sigma_{\mathcal{T}}\right)\right)$ assigning a nonempty set $\operatorname{Mo}_{\mathcal{T}_{\Sigma}}(M)$ of $\Sigma_{\mathcal{T}}$-models to each $\Sigma$-model $M$, such that for every signature morphism $\tau$ : $\Sigma_{1} \rightarrow \Sigma_{2}$ in Sig the following diagram commutes:

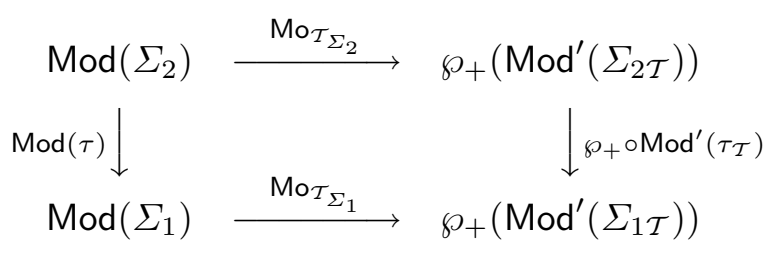

and such that the following satisfaction invariant holds:

$$
\forall \Sigma \in \operatorname{Sig}, \forall E \in \operatorname{Pre}(\Sigma), \forall M \in \operatorname{Mod}(\Sigma): M \vDash E \Leftrightarrow M_{\mathcal{T}} \vDash^{\prime} E_{\mathcal{T}}
$$

where satisfaction is extended to presentations and model sets in the usual way $\left({ }^{4}\right)$, and with henceforth adoption of the following abbreviations:

(a) $E_{\mathcal{T}}$ for $\operatorname{Pr}_{\mathcal{T}_{\Sigma}}(E)$,

(b) $\varphi_{\mathcal{T}}$ for $\{\varphi\}_{\mathcal{T}}$, for a one-sentence presentation $\{\varphi\}$,

(c) $M_{\mathcal{T}}$ for $\mathrm{Mo}_{\mathcal{T}_{\Sigma}}(M)$,

(d) $\vDash$ for $\vDash_{\Sigma}$ and $\vDash^{\prime}$ for $\vDash_{\Sigma_{\mathcal{T}}}^{\prime}$ (and even $\vDash$ for $\vDash^{\prime}$, if no ambiguity arises).

$\left({ }^{4}\right)$ That is, $M \vDash E$ iff $\forall \varphi \in E: M \vDash \varphi, \mathcal{M} \vDash E$ iff $\forall M \in \mathcal{M}: M \vDash E$. 
It may seem strange that presentation transformation is allowed not to respect the set-theoretic structure of presentations, that is, it need not to be constructed elementwise. Our design principle, in this paper as well as in its predecessor [18], is that requirements restrict generality, hence there must be sufficient evidence of their necessity to set them a priori rather than introducing them as properties a posteriori. As an instance of the classical "Ockham's razor", we have adopted the rule: leges non sunt multiplicanda prceter necessitatem.

By our own rule we are thus compelled to argue the necessity of the requirements which form Definition 2.2. Now, requiring functoriality of the signature translation and naturality of the presentation and model transformations is an obvious feature of good structure design by category-theoretic principles. But Definition 2.3 states two further requirements, whose necessity is argued on intuitive grounds as follows.

The intuitive reason for the non-emptiness requirement on $\mathrm{Mo}_{\mathcal{T}_{\Sigma}}(M)$ is that the existence of a pre-institution transformation is intended to entail the "representability" of every source model by some target model.

The reason for the requirement expressed by the satisfaction invariant is the soundness of consequence in the image of the transformation with respect to consequence in the source. This fact is apparent from Proposition 3.3(i), or, more precisely, from the fact that its (very simple, almost immediate) proof requires both directions of the double implication by which the satisfaction invariant is formulated.

The next definition introduces a notion of equivalence between models that reflects their indiscernibility by the logical means that is available "inside the pre-institution", viz. the notion of elementary equivalence. The second part of the definition formalizes logical equivalence of sentences inside a pre-institution, viz. their indiscernibility by validity in models, which fact justifies the overloading of the equivalence symbol.

Definition 2.4. Let $\mathcal{I}=($ Sig, Pre, Mod, $\models)$ be a pre-institution, and $\Sigma$ a signature in Sig.

1. Any two $\Sigma$-models $M_{1}, M_{2}$ are $\mathcal{I}$-equivalent, written $M_{1} \equiv_{\mathcal{I}} M_{2}$, iff for every $\Sigma$-presentation $E$ :

$$
M_{1} \vDash E \Leftrightarrow M_{2} \vDash E .
$$

2. Any two $\Sigma$-sentences $\varphi, \psi$ are $\mathcal{I}$-equivalent, written $\varphi \equiv_{\mathcal{I}} \psi$, iff for every $\Sigma$-model $M$ :

$$
M \vDash \varphi \Leftrightarrow M \vDash \psi .
$$

Additional requirements characterize certain classes of pre-institution transformations, and will prove very useful in the rest of this paper.

Definition 2.5. Let $\mathcal{T}: \mathcal{I} \rightarrow \mathcal{I}^{\prime}$ be a pre-institution transformation, with $\mathcal{I}$, $\mathcal{I}^{\prime}$ as in Definition 2.3. 
(i) $\mathcal{T}$ is adequate iff it meets the following requirement:

$\forall \Sigma \in \operatorname{Sig}, \forall E \in \operatorname{Pre}(\Sigma), \forall M^{\prime} \in \operatorname{Mod}^{\prime}\left(\Sigma_{\mathcal{T}}\right)$ :

$$
M^{\prime} \vDash^{\prime} E_{\mathcal{T}} \Rightarrow \exists M \in \operatorname{Mod}(\Sigma): M^{\prime} \in M_{\mathcal{T}} \wedge M \vDash E .
$$

(ii) $\mathcal{T}$ is fully adequate iff it meets the following requirement: $\forall \Sigma \in \operatorname{Sig}, \forall M^{\prime} \in$ $\operatorname{Mod}^{\prime}\left(\Sigma_{\mathcal{T}}\right)$, for all indexed families $\left\{E_{j}\right\}_{j \in J}$ of $\Sigma$-presentations:

$$
M^{\prime} \vDash^{\prime} \bigcup_{j \in J}\left(E_{j}\right)_{\mathcal{T}} \Rightarrow \exists M \in \operatorname{Mod}(\Sigma): M^{\prime} \in M_{\mathcal{T}} \wedge M \vDash \bigcup_{j \in J} E_{j} .
$$

As we shall see in the next section, adequacy ensures completeness of the transformation with respect to consequence. Full adequacy is just a stronger form of adequacy (clearly, every fully adequate transformation is adequate as well), which proves connected to compactness of pre-institutions, as mentioned in Section 3 and further analysed in Section 4. A useful sufficient criterion for full adequacy is as follows.

LEMMA 2.6. Let $\mathcal{T}: \mathcal{I} \rightarrow \mathcal{I}^{\prime}$ be a pre-institution transformation, with $\mathcal{I}, \mathcal{I}^{\prime}$ as in Definition 2.3. If $\mathcal{T}$ is adequate and meets the following condition:

$\forall \Sigma \in \operatorname{Sig}, \forall M_{1}, M_{2} \in \operatorname{Mod}(\Sigma):\left(M^{\prime} \in M_{1 \mathcal{T}} \wedge M^{\prime} \in M_{2 \mathcal{T}}\right) \Rightarrow M_{1 \mathcal{T}}=M_{2 \mathcal{T}}$, then $\mathcal{T}$ is fully adequate.

A first use of our notions of adequacy is found in the following definition.

Definition 2.7. Let $\mathcal{I}$ and $\mathcal{I}^{\prime}$ be two pre-institutions.

(i) $\mathcal{I}^{\prime}$ is adequately expressive for $\mathcal{I}$, written $\mathcal{I} \preceq \mathcal{I}^{\prime}$, iff there exists an adequate pre-institution transformation $\mathcal{T}: \mathcal{I} \rightarrow \mathcal{I}^{\prime}$.

(ii) $\mathcal{I}^{\prime}$ is fully expressive for $\mathcal{I}$, written $\mathcal{I} \sqsubseteq \mathcal{I}^{\prime}$, iff there exists a fully adequate pre-institution transformation $\mathcal{T}: \mathcal{I} \rightarrow \mathcal{I}^{\prime}$.

Clearly, $\preceq$ and $\sqsubseteq$ are pre-orders, which fact justifies the following

DeFinition 2.8. Let $\mathcal{I}$ and $\mathcal{I}^{\prime}$ be two pre-institutions.

(i) $\mathcal{I}$ and $\mathcal{I}^{\prime}$ have equivalent expressiveness iff $\mathcal{I} \preceq \mathcal{I}^{\prime}$ and $\mathcal{I}^{\prime} \preceq \mathcal{I}$.

(ii) $\mathcal{I}$ and $\mathcal{I}^{\prime}$ have fully equivalent expressiveness iff $\mathcal{I} \sqsubseteq \mathcal{I}^{\prime}$ and $\mathcal{I}^{\prime} \sqsubseteq \mathcal{I}$.

The formal notions of relative expressiveness introduced above are first approximations to an appropriate generalization of classical notions of relative expressiveness of logical systems in the sense of abstract model theory (see [6]). These systems have the limitation of being based on first-order models; as a consequence, also the category of (first-order) signatures is fixed for all logical systems. Our notions are more liberal in that only a functor is required between the signature categories, and model-independence is achieved in a most general manner. To clarify this comparison, we show how a most classical notion of relative expressiveness between logical systems can be captured by a particular transformation of the corresponding pre-institutions. 
EXAMPLE 2.9. If $\mathcal{L}$ is a logical system in the sense of abstract model theory, a corresponding pre-institution $\mathcal{I}_{\mathcal{L}}=(\operatorname{Sig}, \operatorname{Sen}, \operatorname{Mod}, \models)$ is defined as follows: Sig is the category of first-order signatures having only renamings $\left({ }^{5}\right)$ as morphisms; Sen gives for every $\Sigma \in \operatorname{Sig}$ the set of $\Sigma$-sentences in $\mathcal{L}$, and for every renaming $\tau$ : $\Sigma_{1} \rightarrow \Sigma_{2} \in \operatorname{Sig}$ the corresponding translation of $\Sigma_{1}$-sentences into $\Sigma_{2}$-sentences; Mod gives for every $\Sigma \in$ Sig the class of first-order $\Sigma$-models, and for every renaming $\tau: \Sigma_{1} \rightarrow \Sigma_{2} \in$ Sig the corresponding first-order reduction map, which turns each $\Sigma_{2}$-model into a $\Sigma_{1}$-model; finally, satisfaction in $\mathcal{I}_{\mathcal{L}}$ coincides with satisfaction in $\mathcal{L}$.

Now, let $\mathcal{L}, \mathcal{L}^{\prime}$ be logical systems in the sense of abstract model theory. According to [6] (p. 194, Definition 1.2), and [5] (p. 27, Definition 1.1.1), $\mathcal{L}^{\prime}$ is at least as strong as $\mathcal{L}$, which is written $\mathcal{L} \leq \mathcal{L}^{\prime}$, iff for every first-order signature $\Sigma$, for every $\Sigma$-sentence $\varphi$ in $\mathcal{L}$ there is some $\Sigma$-sentence $\psi \in \mathcal{L}^{\prime}$ that has the same models. Let $\mathcal{I}_{\mathcal{L}}, \mathcal{I}_{\mathcal{L}^{\prime}}$ be the pre-institutions that respectively correspond to $\mathcal{L}, \mathcal{L}^{\prime}$. A transformation $\mathcal{T}: \mathcal{I}_{\mathcal{L}} \rightarrow \mathcal{I}_{\mathcal{L}^{\prime}}$ which captures the classical notion of relative expressiveness mentioned above is as follows: whenever $\mathcal{L} \leq \mathcal{L}^{\prime}$, define

$$
\Sigma_{\mathcal{T}}=\Sigma, \quad E_{\mathcal{T}}=\left\{\psi \mid \exists \varphi \in E: \operatorname{Mod}(\varphi)=\operatorname{Mod}^{\prime}(\psi)\right\}, \quad M_{\mathcal{T}}=\{M\} .
$$

It is easy to see (using Lemma 2.6) that $\mathcal{T}$ is a fully adequate transformation.

Besides serving an illustrative purpose, the example also points at the aforementioned target of our present investigation, that is, an appropriate generalization of classical notions of relative expressiveness of logical systems in the sense of abstract model theory.

The classical notion recalled above has classical generalizations that have great methodological importance, and thus high relevance to our investigation. We recall (see Section 3.1 in [5]) that the above notion can also be stated as follows: $\mathcal{L} \leq \mathcal{L}^{\prime}$ iff every elementary class in $\mathcal{L}$ is elementary in $\mathcal{L}^{\prime}$ (where a model class is elementary in $\mathcal{L}$ iff it consists of the models of a sentence in $\mathcal{L}$ ).

A classical generalization of the aforementioned notion of relative expressiveness is the following: $\mathcal{L} \leq_{\mathrm{RPC}} \mathcal{L}^{\prime}$ iff every relativized projective class in $\mathcal{L}$ is a relativized projective class in $\mathcal{L}^{\prime}$ (where a model class is a relativized projective class in $\mathcal{L}$ iff it consists of the relativized $\tau$-reducts of the models of an elementary class in $\mathcal{L}$, for some signature inclusion morphism $\tau\left({ }^{6}\right)$ ).

Now, the main methodological import of the $\leq_{\mathrm{RPC}}$ notion lies not so much in its technical definition as in the reduction scheme that comes along with it, meaning: downward inheritance of model-theoretic properties along the expressiveness ordering. Under the $\leq_{\mathrm{RPC}}$ ordering, the scheme holds for a great variety of model-theoretic properties, including compactness and Löwenheim-Skolem properties - to which we restrict our attention in the present paper.

$\left({ }^{5}\right)$ That is, bijective arity-preserving maps.

$\left.{ }^{6}\right)$ If $\tau: \Sigma_{1} \hookrightarrow \Sigma_{2}$ is a first-order signature inclusion morphism and $M$ is a first-order $\Sigma_{2}$-structure, a relativized $\tau$-reduct of $M$ is any $\Sigma_{1}$-substructure of a $\tau$-reduct of $M$. 
We are thus in presence of a criterion to measure the "appropriateness" of generalizations of classical notions of relative expressiveness to our framework, viz. the extent to which reductions generalize. To be applicable, this criterion preliminarily requires, for each model-theoretic property under consideration, the reformulation of that property within our conceptual framework. Now, in the case of Löwenheim-Skolem properties, we defer this task to Section 7 (as we mentioned in Section 1, a structural enrichment of the notion of pre-institution is necessary). On the contrary, the reformulation of compactness for pre-institutions is fairly obvious, as it appears from the next definition.

Definition 2.10 (Compactness). Let $\mathcal{I}=($ Sig, Sen, Mod, $\vDash)$ be a pre-institution, with Pre $=\wp \circ$ Sen $:$ Sig $\rightarrow$ SET.

(i) $\mathcal{I}$ is compact iff $\forall \Sigma \in \operatorname{Sig}, \forall E \in \operatorname{Pre}(\Sigma): E$ satisfiable $\Leftrightarrow E$ finitely satisfiable.

(ii) $\mathcal{I}$ is consequence-compact iff $\forall \Sigma \in \operatorname{Sig}, \forall E \in \operatorname{Pre}(\Sigma), \forall \varphi \in \operatorname{Sen}(\Sigma)$ :

$$
E \vDash \varphi \Rightarrow \exists F \subseteq E: F \text { finite } \wedge F \vDash \varphi .
$$

R e m ark. The two notions of compactness are equivalent for pre-institutions that are closed under negation (see [6], p. 196, Lemma 2.1), where $\mathcal{I}$ is closed under negation whenever $\forall \Sigma \in \operatorname{Sig}, \forall \varphi \in \operatorname{Sen}(\Sigma), \exists \psi \in \operatorname{Sen}(\Sigma): \forall M \in \operatorname{Mod}(\Sigma)$ : $M \vDash \varphi$ iff not $M \vDash \psi$.

Since finiteness of (sub-)presentations plays an essential rôle in both notions of compactness, one may expect that "suitable" pre-institution transformations for such notions ought to preserve that finiteness somehow. The basic, most intuitive idea is that every sentence should be transformed into a finite set of sentences. This idea is affected by too much of "syntax", though, in the following sense.

If one accepts the abstract model-theoretic purpose proposed in [11], that is "to get away from the syntactic aspects of logic completely and to study classes of structures more in the spirit of universal algebra" then two softenings of the basic idea are in place. First, "finiteness" of the transform $\varphi_{\mathcal{T}}$ of any sentence $\varphi$ should be measured excluding the "tautological" part of $\varphi_{\mathcal{T}}$ ("tautological" relative to $\mathcal{T}$, in a sense made precise below), since model classes are insensitive to tautologies. Second, and more generally in fact, "finiteness" of sentence transformation should only be "up to logical equivalence" in the target pre-institution, since logically equivalent sentences specify identical model classes.

The following definition tells, for a given pre-institution transformation, which sentences of the target pre-institution are "viewed as tautologies" in the source pre-institution; we are thus considering a sort of "stretching" of the classical notion of tautology along the transformation arrow. The subsequent definition, then, formalizes the two "soft" forms of the property we are looking for, according to the rationale given above. 
Definition 2.11. Let $\mathcal{T}: \mathcal{I} \rightarrow \mathcal{I}^{\prime}$ be a pre-institution transformation, with $\mathcal{I}$, $\mathcal{I}^{\prime}$ as in Definition 2.3. Then, for every $\Sigma \in \operatorname{Sig}$ :

(i) a $\Sigma_{\mathcal{T}}$-sentence $\psi$ is a $\mathcal{T}$-tautology iff $\forall M \in \operatorname{Mod}(\Sigma), \forall M^{\prime} \in M_{\mathcal{T}}: M^{\prime} \vDash^{\prime} \psi$,

(ii) $\operatorname{Taut}_{\mathcal{T}}(\Sigma)$ is the set of $\mathcal{T}$-tautologies in $\operatorname{Sen}\left(\Sigma_{\mathcal{T}}\right)$.

Clearly, every $\Sigma_{\mathcal{T}}$-tautology (in the classical sense) is in $\operatorname{Taut}_{\mathcal{T}}(\Sigma)$. This may contain more sentences, however; e.g. if $\emptyset_{\Sigma}$ is the "empty" $\Sigma$-presentation, then clearly $\left(\emptyset_{\Sigma}\right)_{\mathcal{T}} \subseteq \operatorname{Taut}_{\mathcal{T}}(\Sigma)$, and the sentences in $\left(\emptyset_{\Sigma}\right)_{\mathcal{T}}$ need not be $\Sigma_{\mathcal{T}}$ - tautologies.

Definition 2.12. Let $\mathcal{T}: \mathcal{I} \rightarrow \mathcal{I}^{\prime}$ be a pre-institution transformation, with $\mathcal{I}$, $\mathcal{I}^{\prime}$ as in Definition 2.3.

(i) $\mathcal{T}$ is finitary iff $\forall \Sigma \in \operatorname{Sig}, \forall \varphi \in \operatorname{Sen}(\Sigma): \varphi_{\mathcal{T}}-\operatorname{Taut}_{\mathcal{T}}(\Sigma)$ finite.

(ii) $\mathcal{T}$ is quasi-finitary iff $\forall \Sigma \in \operatorname{Sig}, \forall \varphi \in \operatorname{Sen}(\Sigma):\left(\varphi_{\mathcal{T}}-\operatorname{Taut}_{\mathcal{T}}(\Sigma)\right) / \equiv_{\mathcal{I}^{\prime}}$ finite.

The difference between finitarity and quasi-finitarity is illustrated by the transformation in Example 2.9, which is quasi-finitary but not necessarily finitary.

Finally, our last definition relates to equivalence of expressiveness between preinstitutions. If two pre-institutions enjoy equivalent expressiveness, it is sensible to wonder whether the transformations that establish the equivalence are "inverse" to each other in some sense. Among the several possibilities for such a sense, we formalize a notion of equivalence that requires the transformation of logical theories to be the identity; more precisely, the presentation obtained by applying such a transformation and then its inverse to any given presentation is required to have exactly the same consequences as the original presentation. As usual, if $E$ is a $\Sigma$-presentation, $\operatorname{Th}(E)$ denotes the closure of $E$ under consequence, whereas if $M$ is a $\Sigma$-model, then $\operatorname{Th}(M)$ denotes the largest $\Sigma$-presentation that is satisfied by $M$.

Definition 2.13. Let $\mathcal{T}: \mathcal{I} \rightarrow \mathcal{I}^{\prime}$ be a pre-institution transformation, with $\mathcal{I}$, $\mathcal{I}^{\prime}$ as in Definition 2.3. $\mathcal{T}$ is invertible if there exists a pre-institution transformation $\mathcal{R}: \mathcal{I}^{\prime} \rightarrow \mathcal{I}$ such that for every presentation $E$ in $\mathcal{I}: \operatorname{Th}(E)=\operatorname{Th}\left(\left(E_{\mathcal{T}}\right)_{\mathcal{R}}\right)$, in which case $\mathcal{R}$ is termed an inverse of $\mathcal{T}$, and the two pre-institutions $\mathcal{I}, \mathcal{I}^{\prime}$ have exactly equivalent expressiveness.

As a simple illustration, with reference to Example 2.9, it is easily seen that if $\mathcal{L} \leq \mathcal{L}^{\prime}$ and $\mathcal{L}^{\prime} \leq \mathcal{L}$, then the transformation $\mathcal{T}: \mathcal{I}_{\mathcal{L}} \rightarrow \mathcal{I}_{\mathcal{L}^{\prime}}$ is invertible (in fact, it has a fully adequate inverse), i.e. $\mathcal{I}_{\mathcal{L}}$ and $\mathcal{I}_{\mathcal{L}^{\prime}}$ have exactly equivalent expressiveness.

3. Basic facts. In this section, we recall a number of facts and results from [18], which complete the background needed for the further analysis and results presented in this paper. The interested reader is referred to Sections 3 and 4 of [18] for the proofs of the facts recalled here. 
First, noting that we introduced pre-institution transformations as "morphisms", we recall a proposition that justifies that terminology.

Proposition 3.1 (Pre-institution categories). (i) The identity transformation $\mathcal{E}_{\mathcal{I}}: \mathcal{I} \rightarrow \mathcal{I}$ (where $\left.\Sigma_{\mathcal{E}_{\mathcal{I}}}=\Sigma, E_{\mathcal{E}_{\mathcal{I}}}=E, M_{\mathcal{E}_{\mathcal{I}}}=\{M\}\right)$ meets the satisfaction invariant, and is fully adequate.

(ii) If $\mathcal{T}: \mathcal{I} \rightarrow \mathcal{I}^{\prime}$ and $\mathcal{T}^{\prime}: \mathcal{I}^{\prime} \rightarrow \mathcal{I}^{\prime \prime}$ are pre-institution transformations, then so is their composition $\mathcal{T}^{\prime} \circ \mathcal{T}: \mathcal{I} \rightarrow \mathcal{I}^{\prime \prime}$, where:

$$
\begin{aligned}
\Sigma_{\mathcal{T}^{\prime} \circ \mathcal{T}} & =\left(\Sigma_{\mathcal{T}}\right)_{\mathcal{T}^{\prime}}, \\
\tau_{\mathcal{T}^{\prime} \circ \mathcal{T}} & =\left(\tau_{\mathcal{T}}\right)_{\mathcal{T}^{\prime}}, \\
E_{\mathcal{T}^{\prime} \circ \mathcal{T}} & =\left(E_{\mathcal{T}}\right)_{\mathcal{T}^{\prime}}, \\
M_{\mathcal{T}^{\prime} \circ \mathcal{T}} & =\bigcup_{M^{\prime} \in M_{\mathcal{T}}} M_{\mathcal{T}^{\prime}}^{\prime} .
\end{aligned}
$$

(iii) $\mathcal{T}^{\prime} \circ \mathcal{T}$ is adequate if both $\mathcal{T}$ and $\mathcal{T}^{\prime}$ are adequate.

(iv) $\mathcal{T}^{\prime} \circ \mathcal{T}$ is fully adequate if both $\mathcal{T}$ and $\mathcal{T}^{\prime}$ are fully adequate.

(v) Pre-institutions, together with transformations as morphisms, form a category $\mathrm{PT}$, of which a subcategory APT is obtained by taking only adequate transformations as morphisms, of which a subcategory FAPT is obtained by taking only fully adequate transformations as morphisms.

The following fact shows that pre-institution transformations ensure "contravariant" inheritance of the rps and eps properties. This fact seems to be only the first phenomenon of a wealthy situation; the compactness theorem (see below) is another such case. Inheritance is "contravariant" in the sense that, if $\mathcal{T}: \mathcal{I} \rightarrow \mathcal{I}^{\prime}$ is a pre-institution transformation and $\mathcal{I}^{\prime}$ has the property under consideration, then $\mathcal{I}$ has that property as well.

These results demonstrate the usefulness of our notion of transformation, in that they support interesting proof techniques. For example, if a proof of a certain theorem in a pre-institution $\mathcal{I}$ is sought, and the theorem is known to hold in a pre-institution $\mathcal{I}^{\prime}$, it will suffice to find a transformation $\mathcal{T}: \mathcal{I} \rightarrow \mathcal{I}^{\prime}$, since this allows the transfer of the known result back to $\mathcal{I}$.

Another, perhaps more interesting application of these results is concerned with negative results on comparing the expressiveness of pre-institutions, in the sense of Definition 2.7. The proof technique, which has a "contrapositive" flavour, simply consists in showing that some of the properties whose contravariant inheritance is ensured by (possibly "suitable") pre-institution transformations is enjoyed by $\mathcal{I}^{\prime}$ but not by $\mathcal{I}$. In such a case, then, one can infer that no ("suitable") pre-institution transformation $\mathcal{T}: \mathcal{I} \rightarrow \mathcal{I}^{\prime}$ exists (where "suitable" means: with some additional property, such as adequacy). An application of this proof method is presented in Section 5.1 of [18].

Proposition 3.2. Let $\mathcal{T}: \mathcal{I} \rightarrow \mathcal{I}^{\prime}$ be a pre-institution transformation, with $\mathcal{I}, \mathcal{I}^{\prime}$ as in Definition 2.3 . 
(i) If $\mathcal{I}^{\prime}$ is rps, then $\mathcal{I}$ is rps.

(ii) If $\mathcal{I}^{\prime}$ is eps, then $\mathcal{I}$ is eps.

(iii) If $\mathcal{I}^{\prime}$ is ps, then $\mathcal{I}$ is ps.

The reason why (full) adequacy of the transformation is required as a criterion for (full) expressiveness is apparent from the following fact, where $\vDash$ denotes logical consequence, defined in the usual semantical way.

Proposition 3.3. Let $\mathcal{T}: \mathcal{I} \rightarrow \mathcal{I}^{\prime}$ be a pre-institution transformation, with $\mathcal{I}$, $\mathcal{I}^{\prime}$ as in Definition 2.3. Then $\forall \varphi \in \operatorname{Sen}(\Sigma), \forall \psi \in \operatorname{Sen}^{\prime}\left(\Sigma_{\mathcal{T}}\right), \forall E, E_{j} \in \operatorname{Pre}(\Sigma)$ :

(i) $E_{\mathcal{T}} \vDash \varphi_{\mathcal{T}} \Rightarrow E \vDash \varphi$,

(ii) $E \vDash \varphi \Rightarrow E_{\mathcal{T}} \vDash \varphi_{\mathcal{T}}$ if $\mathcal{T}$ is adequate.

(iii) $\bigcup_{j \in J}\left(E_{j}\right)_{\mathcal{T}} \vDash \psi \Rightarrow\left(\bigcup_{j \in J} E_{j}\right)_{\mathcal{T}} \vDash \psi$ if $\mathcal{T}$ is adequate.

(iv) $\left(\bigcup_{j \in J} E_{j}\right)_{\mathcal{T}} \vDash \psi \Rightarrow \bigcup_{j \in J}\left(E_{j}\right)_{\mathcal{T}} \vDash \psi$ if $\mathcal{T}$ is fully adequate.

Furthermore, the following fact $\left({ }^{7}\right)$ tells that, with respect to consequence, $\mathcal{T}$-tautologies behave as tautologies relative to presentation transforms, if the transformation $\mathcal{T}$ is adequate.

Proposition 3.4. Let $\mathcal{T}: \mathcal{I} \rightarrow \mathcal{I}^{\prime}$ be an adequate pre-institution transformation, with $\mathcal{I}, \mathcal{I}^{\prime}$ as in Definition 2.3. Then $\forall \Sigma \in \operatorname{Sig}, \forall E \in \operatorname{Pre}(\Sigma): E_{\mathcal{T}} \vDash$ $\operatorname{Taut}_{\mathcal{T}}(\Sigma)$

Proof. Let $E \in \operatorname{Pre}(\Sigma), M^{\prime} \in \operatorname{Mod}^{\prime}\left(\Sigma_{\mathcal{T}}\right)$, and assume $M^{\prime} \vDash^{\prime} E_{\mathcal{T}}$. By adequacy of $\mathcal{T}$, there exists $M \in \operatorname{Mod}(\Sigma)$ such that $M \vDash E$ and $M^{\prime} \in M_{\mathcal{T}}$. Then $M^{\prime} \vDash^{\prime} \psi$ for every $\psi \in \operatorname{Taut}_{\mathcal{T}}(\Sigma)$, according to Definition 2.11. We conclude that $E_{\mathcal{T}} \vDash$ $\operatorname{Taut}_{\mathcal{T}}(\Sigma)$

The Galois connection nature of invertible pre-institution transformations is revealed by the following characterization.

Proposition 3.5. Let $\mathcal{T}: \mathcal{I} \rightarrow \mathcal{I}^{\prime}$ and $\mathcal{R}: \mathcal{I}^{\prime} \rightarrow \mathcal{I}$ be two pre-institution transformations, with $\mathcal{I}, \mathcal{I}^{\prime}$ as in Definition 2.3. The following conditions are equivalent:

(a) $\mathcal{R}$ is an inverse of $\mathcal{T}$,

(b) $\mathcal{T}$ is an inverse of $\mathcal{R}$,

(c) $\forall \Sigma \in \operatorname{Sig}, \forall E \in \operatorname{Pre}(\Sigma), \forall M^{\prime} \in \operatorname{Mod}\left(\Sigma_{\mathcal{T}}\right): M^{\prime} \vDash^{\prime} E_{\mathcal{T}} \Leftrightarrow\left(M^{\prime}\right)_{\mathcal{R}} \vDash E$,

(d) $\forall \Sigma^{\prime} \in \operatorname{Sig}^{\prime}, \forall E^{\prime} \in \operatorname{Pre}^{\prime}\left(\Sigma^{\prime}\right), \forall M \in \operatorname{Mod}\left(\Sigma_{\mathcal{R}}^{\prime}\right): M \vDash E_{\mathcal{R}}^{\prime} \Leftrightarrow M_{\mathcal{T}} \vDash^{\prime} E^{\prime}$

Sufficient conditions for exactly equivalent expressiveness may be of help in the construction of such equivalences. Of the two conditions given below, the second one is stronger, but may turn out to be more useful in practice.

Proposition 3.6. Let $\mathcal{T}: \mathcal{I} \rightarrow \mathcal{I}^{\prime}$ and $\mathcal{R}: \mathcal{I}^{\prime} \rightarrow \mathcal{I}$ be two pre-institution transformations, with $\mathcal{I}, \mathcal{I}^{\prime}$ as in Definition 2.3. The following conditions are sufficient for $\mathcal{R}$ to be an inverse of $\mathcal{T}$ :

$\left({ }^{7}\right)$ Of which we give the proof here, since this fact was not made explicit in [18]. 
(i) $\forall \Sigma \in \operatorname{Sig}, \forall M \in \operatorname{Mod}(\Sigma), \forall E^{\prime} \in \operatorname{Pre}\left(\Sigma_{\mathcal{T}}\right): M \in\left(M_{\mathcal{T}}\right)_{\mathcal{R}} \wedge\left(M \vDash E_{\mathcal{R}}^{\prime} \Rightarrow\right.$ $\left.M_{\mathcal{T}} \vDash^{\prime} E^{\prime}\right)$

(ii) $\forall \Sigma \in \operatorname{Sig}, \forall M \in \operatorname{Mod}(\Sigma): M \in\left(M_{\mathcal{T}}\right)_{\mathcal{R}} \wedge\left(M_{\mathcal{T}}\right)_{\mathcal{R}} \subseteq \operatorname{Mod}(\operatorname{Th}(M))$.

The conditions in the previous proposition also ensure full adequacy of the inverse transformation, as shown by the following

Proposition 3.7. If $\mathcal{R}: \mathcal{I}^{\prime} \rightarrow \mathcal{I}$ is an inverse of $\mathcal{T}: \mathcal{I} \rightarrow \mathcal{I}^{\prime}$ such that $M \in\left(M_{\mathcal{T}}\right)_{\mathcal{R}}$ holds for every model $M$ in $\mathcal{I}$, then

(i) $\mathcal{R}$ is fully adequate,

(ii) $\mathcal{R} \circ \mathcal{T}$ is fully adequate.

Finally, the following result shows the contravariant inheritance of (two forms of) compactness by suitable pre-institution transformations.

Theorem 3.8 (Compactness). Let $\mathcal{T}: \mathcal{I} \rightarrow \mathcal{I}^{\prime}$ be a fully adequate pre-institution transformation, with $\mathcal{I}, \mathcal{I}^{\prime}$ as in Definition 2.3.

(i) $\mathcal{I}^{\prime}$ compact $\Rightarrow \mathcal{I}$ compact.

(ii) $\left(\mathcal{I}^{\prime}\right.$ consequence-compact $\wedge \mathcal{I}$ quasi-finitary) $\Rightarrow \mathcal{I}$ consequence-compact.

4. Compact transformations. Roughly, a notion of compact transformation is obtained by taking a notion of compactness as introduced for pre-institutions, and "stretching it along the arrow". Thus, compactness (for satisfaction) of a transformation relates satisfiability of a translated presentation to satisfiability of the translation of every finite subpresentation. Similarly, consequencecompactness of a transformation relates consequence from a translated presentation to consequence from the translation of some finite subpresentation. More precisely, we propose the following

Definition 4.1 (Transformation compactness). Let $\mathcal{T}: \mathcal{I} \rightarrow \mathcal{I}^{\prime}$ be a preinstitution transformation, with $\mathcal{I}, \mathcal{I}^{\prime}$ as in Definition 2.3.

(i) $\mathcal{T}$ is compact iff $\forall \Sigma \in \operatorname{Sig}, \forall E \in \operatorname{Pre}(\Sigma):(\forall F \subseteq E: \quad F$ finite $\Rightarrow$ $F_{\mathcal{T}}$ satisfiable) $\Rightarrow E_{\mathcal{T}}$ satisfiable.

(ii) $\mathcal{T}$ is consequence-compact iff $\forall \Sigma \in \operatorname{Sig}, \forall E \in \operatorname{Pre}(\Sigma), \forall \varphi \in \operatorname{Sen}^{\prime}\left(\Sigma_{\mathcal{T}}\right)$ :

$$
E_{\mathcal{T}} \vDash \varphi \Rightarrow \exists F \subseteq E: F \text { finite } \wedge F_{\mathcal{T}} \vDash \varphi .
$$

A number of relationships link compactness of (fully) adequate transformations to compactness of their source and target pre-institutions. These relationships are collected in the following theorem, which subsumes and refines Theorem 3.8 .

Theorem 4.2 (Transformation compactness). Let $\mathcal{T}: \mathcal{I} \rightarrow \mathcal{I}^{\prime}$ be an adequate pre-institution transformation, with $\mathcal{I}, \mathcal{I}^{\prime}$ as in Definition 2.3 .

(i) $\mathcal{T}$ compact $\Leftrightarrow \mathcal{I}$ compact. 
(ii) $\left(\mathcal{T}\right.$ fully adequate $\wedge \mathcal{I}^{\prime}$ compact $) \Rightarrow \mathcal{T}$ compact.

(iii) $(\mathcal{T}$ quasi-finitary $\wedge \mathcal{T}$ consequence-compact $) \Rightarrow \mathcal{I}$ consequence-compact.

(iv) $\left(\mathcal{T}\right.$ fully adequate $\wedge \mathcal{I}^{\prime}$ consequence-compact $) \Rightarrow \mathcal{I}$ consequence-compact.

Proof. (i) $(\Rightarrow)$ Let $E$ be a finitely satisfiable $\Sigma$-presentation in $\mathcal{I}$, thus for every finite $F \subseteq E$ there is a $\Sigma$-model $M_{F}$ such that $M_{F} \vDash F$; then the non-empty $\left(M_{F}\right)_{\mathcal{T}} \vDash^{\prime} F_{\mathcal{T}}$ by the satisfaction invariant, thus compactness of $\mathcal{T}$ entails that $E_{\mathcal{T}}$ is satisfiable, whence satisfiability of $E$ follows from the adequacy of $\mathcal{T}$.

$(\Leftarrow)$ Let $E$ be a $\Sigma$-presentation in $\mathcal{I}$, with $F_{\mathcal{T}}$ satisfiable for every finite $F \subseteq$ $E$; then adequacy of $\mathcal{T}$ entails $F$ satisfiable for every finite $F \subseteq E$, whence $E$ satisfiable follows from compactness of $\mathcal{I}$, and therefore $E_{\mathcal{T}}$ is satisfiable, by the satisfaction invariant.

(ii) Immediate consequence of (i) and Theorem 3.8(i).

(iii) Let $\Sigma \in \operatorname{Sig}, \varphi \in \operatorname{Sen}(\Sigma)$, and $E \in \operatorname{Pre}(\Sigma)$, with $E \vDash \varphi$. Let $F^{\prime}$ be a set consisting of one representative per equivalence class in $\left(\varphi_{\mathcal{T}}-\operatorname{Taut}_{\mathcal{T}}(\Sigma)\right) / \equiv_{\mathcal{I}^{\prime}}$; thus $F^{\prime}$ is finite, since $\mathcal{T}$ is quasi-finitary. Then $E_{\mathcal{T}} \vDash^{\prime} F^{\prime}$, since $E_{\mathcal{T}} \vDash^{\prime} \varphi_{\mathcal{T}}$, which follows from Proposition 3.3(ii) and adequacy of $\mathcal{T}$. Moreover, consequence-compactness of $\mathcal{T}$ entails that for each $\psi \in F^{\prime}$ there is a finite $F_{\psi} \subseteq E$ such that $\left(F_{\psi}\right)_{\mathcal{T}} \vDash^{\prime} \psi$, thus $\bigcup_{\psi \in F^{\prime}}\left(\left(F_{\psi}\right)_{\mathcal{T}}\right) \vDash^{\prime} F^{\prime}$, whence $\left(\bigcup_{\psi \in F^{\prime}} F_{\psi}\right)_{\mathcal{T}} \vDash^{\prime} F^{\prime}$ follows from Proposition 3.3(iii) and adequacy of $\mathcal{T}$.

Let then $F=\bigcup_{\psi \in F^{\prime}} F_{\psi}$. Since $\varphi_{\mathcal{T}} \equiv_{\mathcal{I}^{\prime}} \operatorname{Taut}_{\mathcal{T}}(\Sigma) \cup F^{\prime}$, and $F_{\mathcal{T}} \vDash^{\prime} \operatorname{Taut}_{\mathcal{T}}(\Sigma)$ by Proposition 3.4 and adequacy of $\mathcal{T}$, we infer $F_{\mathcal{T}} \vDash^{\prime} \varphi_{\mathcal{T}}$, whence $F \vDash \varphi$ follows from Proposition 3.3(i). Since $F \subseteq E$ and $F$ is finite, we conclude that $\mathcal{I}$ is consequence-compact.

(iv) Let $\Sigma \in \operatorname{Sig}, \psi \in \operatorname{Sen}^{\prime}\left(\Sigma_{\mathcal{T}}\right)$, and $E \in \operatorname{Pre}(\Sigma)$, with $E_{\mathcal{T}} \vDash^{\prime} \psi$. Since $\mathcal{T}$ is fully adequate, Proposition 3.3(iv) entails $\bigcup_{\varphi \in E} \varphi_{\mathcal{T}} \vDash^{\prime} \psi$. Since $\mathcal{I}^{\prime}$ is consequencecompact, there is a finite $F^{\prime} \subseteq \bigcup_{\varphi \in E} \varphi_{\mathcal{T}}$ such that $F^{\prime} \vDash^{\prime} \psi$. Now, for each $\xi \in F^{\prime}$ pick a $\varphi_{\xi} \in E$ such that $\xi \in\left(\varphi_{\xi}\right)_{\mathcal{T}}$. Then $F^{\prime} \subseteq \bigcup_{\xi \in F^{\prime}}\left(\varphi_{\xi}\right)_{\mathcal{T}}$. Let $F=\left\{\varphi_{\xi} \mid \xi \in\right.$ $\left.F^{\prime}\right\}$. Clearly, $F \subseteq E$ and $F$ finite, and $F^{\prime} \subseteq \bigcup_{\varphi_{\xi} \in F}\left(\varphi_{\xi}\right)_{\mathcal{T}}$, hence $\bigcup_{\varphi_{\xi} \in F}\left(\varphi_{\xi}\right)_{\mathcal{T}} \vDash^{\prime} \psi$. Then, by adequacy of $\mathcal{T}$, Proposition 3.3(iii) entails $F_{\mathcal{T}} \vDash^{\prime} \psi$.

COROLlary 4.3. Theorem 3.8(ii) is an immediate consequence of Theorem 4.2 (iii) and (iv).

Theorem 4.2 thus refines Theorem 3.8 in that it splits the backward inheritance, or "reduction" of compactness, "in two halves": a "source half", whereby the source pre-institution inherits compactness from the transformation, and a "target half", whereby the transformation inherits compactness from the target pre-institution.

The refinement is informative, in that it gives appropriate place to the hypotheses that appear in Theorem 3.8, viz.: 1) the target half of the reduction to full adequacy, for both notions of compactness, and 2) the source half of the reduction to adequacy, for both notions of compactness, and to quasi-finitarity for consequence-compactness. 
The refinement will prove useful in Theorem 6.6, precisely, in Lemma 6.6.12 therein.

5. Abstract sentences, expansion adequacy. We are going to introduce a notion of abstract pre-institution that seems consistent with the abstract modeltheoretic purpose proposed in [11], that we quoted in Section 2 above. Abstraction, in the sense of the following definition, essentially applies to logically equivalent sentences, in the sense that no distinction is drawn between sentences that have exactly the same models.

Definition 5.1 (Abstract pre-institution). A pre-institution $\mathcal{I}=$ (Sig, Sen, Mod, $\models)$ has abstract sentences, or is abstract, iff it meets the following requirement: $\forall \Sigma \in \operatorname{Sig}, \forall \varphi, \psi \in \operatorname{Sen}(\Sigma): \operatorname{Mod}(\varphi)=\operatorname{Mod}(\psi) \Rightarrow \varphi=\psi$.

Abstraction can be applied to every pre-institution that has the ps property by the obvious quotient construction. To each ps pre-institution an abstract form of it corresponds, thus. This is formalized as follows.

Definition 5.2. For each ps pre-institution $\mathcal{I}=($ Sig, Sen, Mod, $\vDash)$, the abstract pre-institution $\widehat{\mathcal{I}}=\left(\operatorname{Sig}, \operatorname{Sen} \widehat{,}, \operatorname{Mod}, \models^{\widehat{*}}\right)$ is defined as having the same category of signatures and model functor, and:

(i) $\forall \Sigma \in \operatorname{Sig}: \operatorname{Sen} \widehat{(}(\Sigma)=\left\{[\varphi]_{\equiv_{\mathcal{I}}} \mid \varphi \in \operatorname{Sen}(\Sigma)\right\}$,

(ii) $\forall \tau: \Sigma_{1} \rightarrow \Sigma_{2} \in \operatorname{Sig}, \forall \varphi \in \operatorname{Sen}\left(\Sigma_{1}\right): \operatorname{Sen} \widehat{\gamma}(\tau)[\varphi]_{\equiv_{\mathcal{I}}}=[\operatorname{Sen}(\tau) \varphi]_{\equiv_{\mathcal{I}}}$,

(iii) $\forall \Sigma \in \operatorname{Sig}, \forall \varphi \in \operatorname{Sen}(\Sigma), \forall M \in \operatorname{Mod}(\Sigma): M \vDash^{\wedge}[\varphi]_{\equiv_{\mathcal{I}}} \Leftrightarrow M \vDash \varphi$.

Clearly, satisfaction in $\widehat{\mathcal{I}}$ is well defined; indeed, satisfaction is invariant under the correspondence established by the previous definition. This entails that the abstraction made by the quotient as in Definition 5.2 gives no loss of information about models, in the sense that elementary equivalence of models is invariant as well.

FACT 5.3. Let $\mathcal{I}$ be a ps pre-institution, with $\widehat{\mathcal{I}}$ the corresponding abstract pre-institution according to Definition 5.2. Then for all models $M_{1}, M_{2}$ in $\mathcal{I}$ (as well as in $\widehat{\mathcal{I}}): M_{1} \equiv_{\widehat{\mathcal{I}}} M_{2} \Leftrightarrow M_{1} \equiv_{\mathcal{I}} M_{2}$.

As an example, we define the abstract, first-order pre-institution, which is relevant to the theorem presented in the next section. The first-order pre-institution is defined according to Example 2.9, except that all first-order signature morphisms are taken, not just the renamings.

EXAMPLE 5.4 (Abstract first-order pre-institution). Let $I=\left(\operatorname{Sig}_{I}, \operatorname{Sen}_{I}\right.$, $\left.\operatorname{Mod}_{I}, \vDash_{I}\right)$ be the first-order pre-institution, with all first-order signature morphisms in $\operatorname{Sig}_{I}$. The abstract first-order pre-institution $\widehat{I}=\left(\operatorname{Sig}_{\hat{I}}, \operatorname{Sen}_{\hat{I}}, \operatorname{Mod}_{\hat{I}}, \vDash_{\hat{I}}\right)$ is defined by $\operatorname{Sig}_{\hat{I}}=\operatorname{Sig}_{I}, \operatorname{Mod}_{\hat{I}}=\operatorname{Mod}_{I}$, and

(i) $\forall \Sigma \in \operatorname{Sig}_{I}: \operatorname{Sen}_{\hat{I}}(\Sigma)=\left\{[\varphi]_{\equiv_{I}} \mid \varphi \in \operatorname{Sen}_{I}(\Sigma)\right\}$,

(ii) $\forall \tau: \Sigma_{1} \rightarrow \Sigma_{2} \in \operatorname{Sig}_{I}, \forall \varphi \in \operatorname{Sen}_{I}\left(\Sigma_{1}\right): \operatorname{Sen}_{\hat{I}}(\tau)[\varphi]_{\equiv_{I}}=\left[\operatorname{Sen}_{I}(\tau) \varphi\right]_{\equiv_{I}}$, 
(iii) $\forall \Sigma \in \operatorname{Sig}_{I}, \forall \varphi \in \operatorname{Sen}_{I}(\Sigma), \forall M \in \operatorname{Mod}_{I}(\Sigma): M \vDash_{\hat{I}}[\varphi]_{\equiv_{I}} \Leftrightarrow M \vDash_{I} \varphi$.

Further, for notational convenience, we extend the use of propositional connectives to the abstract sentences of $\widehat{I}$ by the following convention:

$\neg[\varphi]_{\equiv_{I}}=[\neg \varphi]_{\equiv_{I}}, \quad[\varphi]_{\equiv_{I}} \wedge[\psi]_{\equiv_{I}}=[\varphi \wedge \psi]_{\equiv_{I}}, \quad[\varphi]_{\equiv_{I}} \vee[\psi]_{\equiv_{I}}=[\varphi \vee \psi]_{\equiv_{I}}$.

As one should expect from Definition 2.12, turning sentences into abstract ones has the effect of turning quasi-finitary pre-institution transformations into finitary ones.

FACT 5.5. If $\mathcal{T}: \mathcal{I} \rightarrow \mathcal{I}^{\prime}$ is a quasi-finitary pre-institution transformation, with $\mathcal{I}, \mathcal{I}^{\prime}$ as in Definition 2.3 , then $\widehat{\mathcal{I}}: \mathcal{I} \rightarrow \widehat{\mathcal{I}}^{\prime}$ defined by

(i) $\forall \Sigma \in$ Sig: $\Sigma_{\widehat{\mathcal{T}}}=\Sigma_{\mathcal{T}}$,

(ii) $\forall \Sigma \in \operatorname{Sig}, \forall E \in \operatorname{Pre}(\Sigma): E_{\widehat{\mathcal{T}}}=\left\{[\varphi]_{\equiv_{\mathcal{I}^{\prime}}} \mid \varphi \in E_{\mathcal{T}}\right\}$,

(iii) $\forall \Sigma \in \operatorname{Sig}, \forall M \in \operatorname{Mod}(\Sigma): M_{\widehat{\mathcal{T}}}=M_{\mathcal{T}}$

is a finitary pre-institution transformation.

The identification of sentences up to logical equivalence has some technical convenience, which will surface in the proof of the theorem presented in the next section. In the formulation of that theorem, yet another property of pre-institution transformations is needed, which mirrors the notion of adequacy, but applied to model expansions rather than to models.

Definition 5.6 (Expansion adequacy). Let $\mathcal{T}: \mathcal{I} \rightarrow \mathcal{I}^{\prime}$ be a pre-institution transformation, with $\mathcal{I}, \mathcal{I}^{\prime}$ as in Definition 2.3. $\mathcal{T}$ is expansion-adequate iff $\forall \tau$ : $\Sigma_{1} \rightarrow \Sigma_{2} \in \operatorname{Sig}, \forall M_{1} \in \operatorname{Mod}\left(\Sigma_{1}\right), \forall M_{2}^{\prime} \in \operatorname{Mod}^{\prime}\left(\Sigma_{2} \mathcal{T}\right):$

$$
M_{2}^{\prime} \tau_{\mathcal{T}} \in M_{1 \mathcal{T}} \Rightarrow \exists M_{2} \in \operatorname{Mod}\left(\Sigma_{2}\right): M_{2} \tau=M_{1} \wedge M_{2}^{\prime} \in M_{2 \mathcal{T}} .
$$

In other words, $\mathcal{T}$ is expansion-adequate whenever every $\tau_{\mathcal{T}}$-expansion of a transform of any given model is a transform of a $\tau$-expansion of that model, for all signature morphisms $\tau$ in the source pre-institution.

6. Equivalence with the abstract, first-order pre-institution. We start with a couple of general definitions and propositions, relating to discernibility of models that are transforms of the same model, along a given pre-institution transformation.

DeFINITION 6.1. Let $\mathcal{T}: \mathcal{I} \rightarrow \mathcal{I}^{\prime}$ be a pre-institution transformation, with $\mathcal{I}$, $\mathcal{I}^{\prime}$ as in Definition 2.3. $\mathcal{T}$ is $\equiv_{\mathcal{I}^{-} \text {-limited }}$ iff $\forall \Sigma \in \operatorname{Sig}, \forall \varphi \in \operatorname{Sen}(\Sigma), \forall M \in \operatorname{Mod}(\Sigma)$, $\forall M_{1}^{\prime}, M_{2}^{\prime} \in M_{\mathcal{T}}: M_{1}^{\prime} \vDash^{\prime} \varphi_{\mathcal{T}} \Leftrightarrow M_{2}^{\prime} \vDash^{\prime} \varphi_{\mathcal{T}}$.

Definition 6.2. Let $\mathcal{I}: \mathcal{I} \rightarrow \mathcal{I}^{\prime}$ be a pre-institution transformation, with $\mathcal{I}, \mathcal{I}^{\prime}$ as in Definition 2.3. $\mathcal{T}$ is $\equiv_{\mathcal{I}^{\prime}}$-limited iff $\forall \Sigma \in \operatorname{Sig}, \forall M \in \operatorname{Mod}(\Sigma), \forall M_{1}^{\prime}, M_{2}^{\prime} \in M_{\mathcal{T}}$ : $M_{1}^{\prime} \equiv_{\mathcal{I}^{\prime}} M_{2}^{\prime}$.

Of the two properties introduced above, the latter entails the former, whilst models that are transforms of the same model along a $\equiv_{\mathcal{I}}$-limited transformation 
$\mathcal{T}: \mathcal{I} \rightarrow \mathcal{I}^{\prime}$ may well be discernible by sentences of the target pre-institutionalbeit not by translations of sentences of the source pre-institution.

FACT 6.3. If $\mathcal{T}: \mathcal{I} \rightarrow \mathcal{I}^{\prime}$ is a $\equiv_{\mathcal{I}^{\prime}}$-limited pre-institution transformation, then it is $\equiv_{\mathcal{I}}$-limited.

Proof. Immediate.

Both of the following facts play a rôle in the subsequent, main theorem.

Proposition 6.4. If the pre-institution $\mathcal{I}$ is closed under negation, then every fully adequate transformation $\mathcal{T}: \mathcal{I} \rightarrow \mathcal{I}^{\prime}$ is $\equiv_{\mathcal{I}}$-limited.

Proof. By contradiction, assume that for some $\Sigma$-sentence $\varphi$ and $\Sigma$-model $M$ in $\mathcal{I}$ there exist $M_{1}^{\prime}$ and $M_{2}^{\prime}$, both in $M_{\mathcal{T}}$, such that $M_{1}^{\prime} \vDash^{\prime} \varphi_{\mathcal{T}}$ and not $M_{2}^{\prime} \vDash^{\prime} \varphi_{\mathcal{T}}$. Then not $M_{\mathcal{T}} \vDash^{\prime} \varphi_{\mathcal{T}}$, thus not $M \vDash \varphi$ by the satisfaction invariant, that is $M \vDash \neg \varphi$, whence $M_{1}^{\prime} \vDash^{\prime}(\neg \varphi)_{\mathcal{T}}$; therefore $M_{1}^{\prime} \vDash^{\prime} \varphi_{\mathcal{T}} \cup(\neg \varphi)_{\mathcal{T}}$, whence by full adequacy of $\mathcal{T}, \exists M_{1}: M_{1}^{\prime} \in M_{1 \mathcal{T}} \wedge M_{1} \vDash\{\varphi\} \cup\{\neg \varphi\}$, i.e. $M_{1} \vDash \varphi$ and not $M_{1} \vDash \varphi$, which is absurd.

Proposition 6.5. Let $\mathcal{T}: \mathcal{I} \rightarrow \mathcal{I}^{\prime}$ be a pre-institution transformation. If $\mathcal{T}$ is $\equiv_{\mathcal{I}}$-limited, then $M_{1 \mathcal{T}} \cap M_{2 \mathcal{T}} \neq\{\} \Rightarrow M_{1} \equiv_{\mathcal{I}} M_{2}$ holds for all models $M_{1}$, $M_{2}$ in $\mathcal{I}$.

Proof. For all sentences $\varphi$ and models $M$ in $\mathcal{I}$, Definition 6.1 and the satisfaction invariant entail that $\forall M^{\prime} \in M_{\mathcal{T}}: M^{\prime} \vDash^{\prime} \varphi_{\mathcal{T}} \Leftrightarrow M_{\mathcal{T}} \vDash^{\prime} \varphi_{\mathcal{T}} \Leftrightarrow M \vDash \varphi$. Thus $M^{\prime} \in M_{1 \mathcal{T}} \cap M_{2 \mathcal{T}}$ implies that for every sentence $\varphi$ in $\mathcal{I}: M^{\prime} \vDash^{\prime} \varphi_{\mathcal{T}} \Leftrightarrow$ $M_{1} \vDash \varphi \Leftrightarrow M_{2} \vDash \varphi$, i.e. $M_{1} \equiv_{\mathcal{I}} M_{2}$.

The idea for the following theorem comes from Lemma XII.2.3 of [6], which plays a significant rôle in the proof of the first Lindström theorem as presented in [6]. The lemma says the following. Let $\mathcal{L}_{I}$ be the first-order logical system (in the sense of abstract model theory), and $\mathcal{L}$ a compact regular $\left(^{8}\right)$ logical system such that $\mathcal{L}_{I} \leq \mathcal{L}$. If $M_{1} \equiv_{\mathcal{L}_{I}} M_{2} \Rightarrow M_{1} \equiv_{\mathcal{L}} M_{2}$ holds for all first-order models $M_{1}, M_{2}$, then $\mathcal{L} \leq \mathcal{L}_{I}$. Now, the following theorem gives a similar result in our abstract framework, but without asking any regularity condition. On the other hand, the theorem is not-properly speaking - a generalization of the recalled lemma, for only one reason: it only applies to abstract pre-institutions. However, as we argue at the end of this section, a slight variant of the theorem holds that is a proper generalization of the recalled lemma.

THEOREM 6.6 (Equivalence with the abstract first-order pre-institution). Let $\widehat{I}$ be the abstract first-order pre-institution, with $\widehat{I}=\left(\operatorname{Sig}_{\hat{I}}, \operatorname{Sen}_{\hat{I}}, \operatorname{Mod}_{\hat{I}}, \vDash_{\hat{I}}\right)$ according to Example 5.4, and $\mathcal{L}=\left(\operatorname{Sig}_{\mathcal{L}}, \operatorname{Sen}_{\mathcal{L}}, \operatorname{Mod}_{\mathcal{L}}, \vDash_{\mathcal{L}}\right)$ an abstract pre-institu-

$\left({ }^{8}\right)$ This means that $\mathcal{L}$ is closed under propositional connectives, permits relativization, and allows elimination of function symbols and constants - see Definition XII.1.3 in [6] for further details. 
tion that is consequence-compact and has the ps property. If there exists a transformation $\mathcal{T}: \widehat{I} \rightarrow \mathcal{L}$ such that:

(i) $\mathrm{Si}_{\mathcal{T}}: \operatorname{Sig}_{\hat{I}} \rightarrow \operatorname{Sig}_{\mathcal{L}}$ is an isomorphism of categories;

(ii) $\mathcal{T}$ is fully adequate and expansion-adequate;

(iii) $\forall \Sigma \in \operatorname{Sig}_{\hat{I}}, \forall M^{\prime} \in \operatorname{Mod}_{\mathcal{L}}\left(\Sigma_{\mathcal{T}}\right): \exists M \in \operatorname{Mod}_{\hat{I}}(\Sigma): M^{\prime} \in M_{\mathcal{T}}$;

(iv) $\forall \Sigma \in \operatorname{Sig}_{\hat{I}}, \forall M_{1}, M_{2} \in \operatorname{Mod}_{\hat{I}}(\Sigma), \forall M_{1}^{\prime} \in M_{1 \mathcal{T}}, \forall M_{2}^{\prime} \in M_{2 \mathcal{T}}: \quad M_{1} \equiv_{\hat{I}} M_{2}$ $\Rightarrow M_{1}^{\prime} \equiv_{\mathcal{L}} M_{2}^{\prime}$,

then $\mathcal{T}$ has a fully adequate, expansion-adequate and finitary inverse.

Proof. For the sake of conciseness and modularity, the proof is structured into a number of local definitions and lemmas, which are to be understood under the assumptions and with the notation that are introduced by the hypotheses of the theorem (we contribute this somewhat unusual proof style to the investigation on the engineering of mathematical arguments - cf. [8]).

DeFinition 6.6.1. $\operatorname{Si}_{\mathcal{R}}: \operatorname{Sig}_{\mathcal{L}} \rightarrow \operatorname{Sig}_{\hat{I}} \stackrel{\text { def }}{=}\left(\operatorname{Si}_{\mathcal{T}}\right)^{-1}$.

Definition 6.6.2. (i) If $M^{\prime} \in \operatorname{Mod}_{\mathcal{L}}\left(\Sigma^{\prime}\right)$ for some $\Sigma^{\prime} \in \operatorname{Sig}_{\mathcal{L}}$, then $M_{\mathcal{R}}^{\prime} \stackrel{\text { def }}{=}$ $\left\{M \mid M^{\prime} \in M_{\mathcal{T}}\right\}$.

(ii) If $\mathcal{M}^{\prime} \subseteq \operatorname{Mod}_{\mathcal{L}}\left(\Sigma^{\prime}\right)$ for some $\Sigma^{\prime} \in \operatorname{Sig}_{\mathcal{L}}$, then $\mathcal{M}_{\mathcal{R}}^{\prime} \stackrel{\text { def }}{=} \bigcup_{M^{\prime} \in \mathcal{M}^{\prime}} M_{\mathcal{R}}^{\prime}$.

Lemma 6.6.3. $\left(M^{\prime}\right)_{\mathcal{R}} \neq\{\}$ for every model $M^{\prime}$ in $\mathcal{L}$

Proof. By hypothesis (iii), thanks to Definition 6.6.2(i).

Lemma 6.6.4. $M^{\prime} \in\left(M_{\mathcal{R}}^{\prime}\right)_{\mathcal{T}}$ for every model $M^{\prime}$ in $\mathcal{L}$.

Proof. Definition 6.6.2(i) entails $\forall M \in M_{\mathcal{R}}^{\prime}: M^{\prime} \in M_{\mathcal{T}}$, and according to Lemma 6.6.3, $\exists M \in M_{\mathcal{R}}^{\prime}$; thus $\exists M \in M_{\mathcal{R}}^{\prime}: M^{\prime} \in M_{\mathcal{T}}$, which is equivalent to $M^{\prime} \in\left(M_{\mathcal{R}}^{\prime}\right)_{\mathcal{T}}$.

Lemma 6.6.5. $M \in\left(M_{\mathcal{T}}\right)_{\mathcal{R}}$ for every model $M$ in $\widehat{I}$.

P r o of. Definition 6.6.2(i) entails $\forall M^{\prime} \in M_{\mathcal{T}}: M \in\left(M^{\prime}\right)_{\mathcal{R}}$, and by hypothesis $\exists M^{\prime} \in M_{\mathcal{T}}$ (since $\mathcal{T}$ is a pre-institution transformation); thus $\exists M^{\prime} \in M_{\mathcal{T}}: M \in$ $\left(M^{\prime}\right)_{\mathcal{R}}$, i.e. $M \in\left(M_{\mathcal{T}}\right)_{\mathcal{R}}$, by Definition 6.6.2(ii).

Lemma 6.6.6. $\mathcal{T}$ is $\equiv_{\mathcal{L}}$-limited.

Proof. Follows from hypothesis (iv) and Definition 6.2 , since $M \equiv_{\hat{I}} M$ for all first-order models $M$.

Lemma 6.6.7. $\forall M_{1}, M_{2} \in M_{\mathcal{R}}^{\prime}: M_{1} \equiv_{\hat{I}} M_{2}$, for every model $M^{\prime}$ in $\mathcal{L}$.

Proof. If both $M_{1}$ and $M_{2}$ are in $M_{\mathcal{R}}^{\prime}$, then $M^{\prime} \in M_{1 \mathcal{T}} \cap M_{2 \mathcal{T}}$ by Definition 6.6.2(i), thus $M_{1 \mathcal{T}} \cap M_{2 \mathcal{T}} \neq\{\}$, whence $M_{1} \equiv_{\hat{I}} M_{2}$ follows from Lemma 6.6.6, Fact 6.3 and Proposition 6.5.

As a standard consequence of the elementary equivalence of all models in $M_{\mathcal{R}}^{\prime}$ we obtain 
CoROLlaRY 6.6.8. $\mathrm{Th}_{I}\left(M_{\mathcal{R}}^{\prime}\right)$ is a complete first-order theory, for every model $M^{\prime}$ in $\mathcal{L}$.

Lemma 6.6.9. $\forall \tau^{\prime}: \Sigma_{1}^{\prime} \rightarrow \Sigma_{2}^{\prime} \in \operatorname{Sig}_{\mathcal{L}}, \forall M^{\prime} \in \operatorname{Mod}_{\mathcal{L}}\left(\Sigma_{2}^{\prime}\right): M_{\mathcal{R}}^{\prime} \tau_{\mathcal{R}}^{\prime}=\left(M^{\prime} \tau^{\prime}\right)_{\mathcal{R}}$.

Pro of. We show that (a) $M_{\mathcal{R}}^{\prime} \tau_{\mathcal{R}}^{\prime} \subseteq\left(M^{\prime} \tau^{\prime}\right)_{\mathcal{R}}$, and (b) $\left(M^{\prime} \tau^{\prime}\right)_{\mathcal{R}} \subseteq M_{\mathcal{R}}^{\prime} \tau_{\mathcal{R}}^{\prime}$.

(a) Let $M_{1} \in M_{\mathcal{R}}^{\prime} \tau_{\mathcal{R}}^{\prime}$. Then $\exists M_{2} \in M_{\mathcal{R}}^{\prime}: M_{1}=M_{2} \tau_{\mathcal{R}}^{\prime}$, hence $\exists M_{2}: M_{1}=$ $M_{2} \tau_{\mathcal{R}}^{\prime} \wedge M^{\prime} \in M_{2 \mathcal{T}}$, by Definition 6.6.2(i). Then $M^{\prime} \tau^{\prime} \in M_{2 \mathcal{T}} \tau^{\prime}=\left(M_{2} \tau_{\mathcal{R}}^{\prime}\right)_{\mathcal{T}}$ by naturality of model transformation in $\mathcal{T}$ and hypothesis (i), and since $M_{1}=$ $M_{2} \tau_{\mathcal{R}}^{\prime}$, we can infer $M^{\prime} \tau^{\prime} \in M_{1 \mathcal{T}}$, whence $M_{1} \in\left(M^{\prime} \tau^{\prime}\right)_{\mathcal{R}}$ by Definition 6.6.2(i).

(b) Let $M_{1} \in\left(M^{\prime} \tau^{\prime}\right)_{\mathcal{R}}$. Then $M^{\prime} \tau^{\prime} \in M_{1 \mathcal{T}}$ by Definition 6.6.2(i), whence hypothesis (i) and expansion-adequacy of $\mathcal{T}$ ensure that $\exists M_{2}: M_{1}=M_{2} \tau_{\mathcal{R}}^{\prime} \wedge$ $M^{\prime} \in M_{2 \mathcal{T}}$. Definition 6.6.2(i) then entails $M_{2} \in M_{\mathcal{R}}^{\prime}$, thus $M_{2} \tau_{\mathcal{R}}^{\prime} \in M_{\mathcal{R}}^{\prime} \tau_{\mathcal{R}}^{\prime}$, i.e. $M_{1} \in M_{\mathcal{R}}^{\prime} \tau_{\mathcal{R}}^{\prime}$

Lemma 6.6.10. $\forall \Sigma \in \operatorname{Sig}_{\hat{I}}, \forall \varphi \in \operatorname{Sen}_{\hat{I}}(\Sigma), \forall M^{\prime} \in \operatorname{Mod}_{\mathcal{L}}\left(\Sigma_{\mathcal{T}}\right): M_{\mathcal{R}}^{\prime} \vDash_{\hat{I}} \varphi \Leftrightarrow$ $M^{\prime} \vDash_{\mathcal{L}} \varphi_{\mathcal{T}}$.

Proof. Let $\Sigma \in \operatorname{Sig}_{\hat{I}}, \varphi \in \operatorname{Sen}_{\hat{I}}(\Sigma)$, and $M^{\prime} \in \operatorname{Mod}_{\mathcal{L}}\left(\Sigma_{\mathcal{T}}\right)$. Then

$$
\begin{aligned}
& M_{\mathcal{R}}^{\prime} \vDash_{\hat{I}} \varphi \\
& \quad \Leftrightarrow \forall M \in M_{\mathcal{R}}^{\prime}: M \vDash_{\hat{I}} \varphi \\
& \Leftrightarrow \forall M \in \operatorname{Mod}_{\hat{I}}(\Sigma): M^{\prime} \in M_{\mathcal{T}} \Rightarrow M \vDash_{\hat{I}} \varphi \text { (by Definition 6.6.2(i)) } \\
& \Leftrightarrow \forall M \in \operatorname{Mod}_{\hat{I}}(\Sigma): M^{\prime} \in M_{\mathcal{T}} \Rightarrow M_{\mathcal{T}} \vDash_{\mathcal{L}} \varphi_{\mathcal{T}} \text { (by the satisfaction invariant) } \\
& \quad \Leftrightarrow M^{\prime} \vDash_{\mathcal{L}} \varphi_{\mathcal{T}} \text { (by Lemma } 6.6 .6 \text { and Definition 6.2). - }
\end{aligned}
$$

Lemma 6.6.11. $\forall \Sigma^{\prime} \in \operatorname{Sig}_{\mathcal{L}}, \forall \psi \in \operatorname{Sen}_{\mathcal{L}}\left(\Sigma^{\prime}\right), \forall M^{\prime} \in \operatorname{Mod}_{\mathcal{L}}\left(\Sigma^{\prime}\right): M^{\prime} \vDash_{\mathcal{L}} \psi \Rightarrow$ $\left(\operatorname{Th}_{\hat{I}}\left(M_{\mathcal{R}}^{\prime}\right)\right)_{\mathcal{T}} \vDash_{\mathcal{L}} \psi$.

Proof. Assume $M^{\prime} \vDash_{\mathcal{L}} \psi$, and let $N^{\prime} \vDash_{\mathcal{L}}\left(\operatorname{Th}_{\hat{I}}\left(M_{\mathcal{R}}^{\prime}\right)\right)_{\mathcal{T}}$. By adequacy of $\mathcal{T}, \exists M \in \operatorname{Mod}_{\hat{I}}\left(\Sigma_{\mathcal{R}}^{\prime}\right): N^{\prime} \in M_{\mathcal{T}} \wedge M \vDash_{\hat{I}} \operatorname{Th}_{\hat{I}}\left(M_{\mathcal{R}}^{\prime}\right)$. Then $\operatorname{Th}_{\hat{I}}\left(M_{\mathcal{R}}^{\prime}\right)=\operatorname{Th}_{\hat{I}}(M)$ by Corollary 6.6.8, and $M \in N_{\mathcal{R}}^{\prime}$ by Definition 6.6.2(i). Therefore $\operatorname{Th}_{\hat{I}}\left(N_{\mathcal{R}}^{\prime}\right)=$ $\operatorname{Th}_{\hat{I}}\left(M_{\mathcal{R}}^{\prime}\right)$ by Lemma 6.6.7, whence $\forall M \in M_{\mathcal{R}}^{\prime}, \forall N \in N_{\mathcal{R}}^{\prime}: M \equiv_{\hat{I}} N$. Then hypothesis (iv) and Definition 6.6.2(i) entail $M^{\prime} \equiv_{\mathcal{L}} N^{\prime}$, whence $N^{\prime} \vDash_{\mathcal{L}} \psi$ follows from the assumption.

Lemma 6.6.12. $\forall \Sigma^{\prime} \in \operatorname{Sig}_{\mathcal{L}}, \forall \psi \in \operatorname{Sen}_{\mathcal{L}}\left(\Sigma^{\prime}\right), \forall M^{\prime} \in \operatorname{Mod}_{\mathcal{L}}\left(\Sigma^{\prime}\right):$

$$
M^{\prime} \vDash_{\mathcal{L}} \psi \Rightarrow \exists \varphi \in \operatorname{Sen}_{\hat{I}}: M_{\mathcal{R}}^{\prime} \vDash_{\hat{I}} \varphi \wedge \varphi_{\mathcal{T}} \vDash_{\mathcal{L}} \psi .
$$

Proof. If $M^{\prime} \vDash_{\mathcal{L}} \psi$, then $\left(\operatorname{Th}_{\hat{I}}\left(M_{\mathcal{R}}^{\prime}\right)\right)_{\mathcal{T}} \vDash_{\mathcal{L}} \psi$ by Lemma 6.6.11. Moreover, $\mathcal{T}$ is consequence-compact by Theorem 4.2(iv), since by hypothesis $\mathcal{L}$ is consequencecompact and $\mathcal{T}$ is fully adequate. There exists thus a finite $F=\left\{\widehat{\varphi}_{1}, \ldots, \widehat{\varphi}_{n}\right\} \subseteq$ $\operatorname{Th}_{\hat{I}}\left(M_{\mathcal{R}}^{\prime}\right)$ such that $F_{\mathcal{T}} \vDash_{\mathcal{L}} \psi$, where $\varphi_{1}, \ldots, \varphi_{n}$ are first-order sentences; let then $\varphi=\left[\varphi_{1} \wedge \ldots \wedge \varphi_{n}\right]_{\equiv_{I}}$. Clearly $F \equiv_{\hat{I}}\{\varphi\}$, hence $F_{\mathcal{T}} \equiv_{\mathcal{L}} \varphi_{\mathcal{T}}$ by adequacy of $\mathcal{T}$ and Proposition 3.3(ii), whence $\varphi_{\mathcal{T}} \vDash_{\mathcal{L}} \psi$. 
Lemma 6.6.13. If $\Sigma^{\prime} \in \operatorname{Sig}_{\mathcal{L}}$ and $\psi \in \operatorname{Sen}_{\mathcal{L}}\left(\Sigma^{\prime}\right)$, for each $M^{\prime} \in \operatorname{Mod}_{\mathcal{L}}(\psi)$ let $\varphi_{M^{\prime}}$ be some sentence in $\operatorname{Sen}_{\hat{I}}\left(\Sigma_{\mathcal{R}}^{\prime}\right)$ such that $M_{\mathcal{R}}^{\prime} \vDash_{\hat{I}} \varphi_{M^{\prime}} \wedge\left(\varphi_{M^{\prime}}\right)_{\mathcal{T}} \vDash_{\mathcal{L}} \psi$ (such a sentence exists according to Lemma 6.6.12). Then we have $\operatorname{Mod}_{\mathcal{L}}(\psi)=$ $\bigcup_{M^{\prime} \in \operatorname{Mod}_{\mathcal{L}}(\psi)} \operatorname{Mod}_{\mathcal{L}}\left(\left(\varphi_{M^{\prime}}\right)_{\mathcal{T}}\right)$.

Pro of. If $M^{\prime} \in \operatorname{Mod}_{\mathcal{L}}(\psi)$, then $M_{\mathcal{R}}^{\prime} \vDash_{\hat{I}} \varphi_{M^{\prime}}$ by hypothesis, hence $\left(M_{\mathcal{R}}^{\prime}\right)_{\mathcal{T}} \vDash_{\mathcal{L}}$ $\left(\varphi_{M^{\prime}}\right)_{\mathcal{T}}$ by the satisfaction invariant, whence $M^{\prime} \vDash_{\mathcal{L}}\left(\varphi_{M^{\prime}}\right)_{\mathcal{T}}$ follows from Lemma 6.6.4.

Conversely, if $M^{\prime} \vDash_{\mathcal{L}}\left(\varphi_{N^{\prime}}\right)_{\mathcal{T}}$ for some $N^{\prime} \in \operatorname{Mod}_{\mathcal{L}}(\psi)$, then $M^{\prime} \vDash_{\mathcal{L}} \psi$ follows from Lemma 6.6.12, since $\left(\varphi_{N^{\prime}}\right)_{\mathcal{T}} \vDash_{\mathcal{L}} \psi$.

Lemma 6.6.14. If $\Sigma^{\prime} \in \operatorname{Sig}_{\mathcal{L}}$ and $\psi \in \operatorname{Sen}_{\mathcal{L}}\left(\Sigma^{\prime}\right)$, for each $M^{\prime} \in \operatorname{Mod}_{\mathcal{L}}(\psi)$ let $\varphi_{M^{\prime}}$ be as in Lemma 6.6.13. Then there exists a finite $\mathcal{M}^{\prime} \subseteq \operatorname{Mod}_{\mathcal{L}}(\psi)$ such that $\operatorname{Mod}_{\mathcal{L}}(\psi)=\bigcup_{M^{\prime} \in \mathcal{M}^{\prime}} \operatorname{Mod}_{\mathcal{L}}\left(\left(\varphi_{M^{\prime}}\right)_{\mathcal{T}}\right)$.

Pro of. Assume, by contradiction, that for every finite $\mathcal{M}^{\prime} \subseteq \operatorname{Mod}_{\mathcal{L}}(\psi)$ there exists $N^{\prime} \in \operatorname{Mod}_{\mathcal{L}}(\psi)$ such that $\forall M^{\prime} \in \mathcal{M}^{\prime}$ : not $N^{\prime} \vDash_{\mathcal{L}}\left(\varphi_{M^{\prime}}\right)_{\mathcal{T}}$, thus also $N_{\mathcal{R}}^{\prime} \vDash_{\hat{I}} \neg \varphi_{M^{\prime}}$ by Lemma 6.6.10 and Corollary 6.6.8, therefore $N^{\prime} \vDash_{\mathcal{L}}\{\psi\} \cup$ $\bigcup_{M^{\prime} \in \mathcal{M}^{\prime}}\left(\left(\neg \varphi_{M^{\prime}}\right) \mathcal{T}\right)$ by the satisfaction invariant and Lemma 6.6.4. This entails that, for every finite $F \subseteq\left\{\neg \varphi_{M^{\prime}} \mid M^{\prime} \in \operatorname{Mod}_{\mathcal{L}}(\psi)\right\}$, the set $\{\psi\} \cup \bigcup_{\varphi \in F} \varphi_{\mathcal{T}}$ is satisfiable. From this fact, satisfiability of every finite $F^{\prime} \subseteq\{\psi\} \cup$ $\bigcup_{M^{\prime} \in \operatorname{Mod}_{\mathcal{L}}(\psi)}\left(\left(\neg \varphi_{M^{\prime}}\right)_{\mathcal{T}}\right)$ can be inferred as follows. For each $\chi \in F^{\prime}-\{\psi\}$, pick an $M_{\chi}^{\prime}$ such that $\chi \in\left(\neg \varphi_{M_{\chi}^{\prime}}\right)_{\mathcal{T}}$. Let $G^{\prime}=\{\psi\} \cup \bigcup_{\chi \in F^{\prime}-\{\psi\}}\left(\left(\neg \varphi_{M_{\chi}^{\prime}}\right)_{\mathcal{T}}\right)$. Since the set $G=\left\{\neg \varphi_{M_{\chi}^{\prime}} \mid \chi \in F^{\prime}-\{\psi\}\right\}$ is finite, and moreover $G \subseteq\left\{\neg \varphi_{M^{\prime}} \mid\right.$ $\left.M^{\prime} \in \operatorname{Mod}_{\mathcal{L}}(\psi)\right\}$, the set $G^{\prime}$ is satisfiable, as shown above, hence so is every finite subset of $G^{\prime}$. Since every finite $F^{\prime}$ as above is a subset of some such $G^{\prime}$, it follows that every such $F^{\prime}$ is satisfiable as well. From compactness of $\mathcal{L}$ we then infer satisfiability of $\{\psi\} \cup \bigcup_{M^{\prime} \in \operatorname{Mod}_{\mathcal{L}}(\psi)}\left(\left(\neg \varphi_{M^{\prime}}\right)_{\mathcal{T}}\right)$, i.e. existence of an $M^{\prime} \in \operatorname{Mod}_{\mathcal{L}}(\psi)$ such that $M^{\prime} \vDash_{\mathcal{L}}\left(\neg \varphi_{M^{\prime}}\right)_{\mathcal{T}}$, which implies $M_{\mathcal{R}}^{\prime} \vDash_{\hat{I}} \neg \varphi_{M^{\prime}}$ by Lemma 6.6 .10 , hence not $M_{\mathcal{R}}^{\prime} \vDash_{\hat{I}} \varphi_{M^{\prime}}$, by Corollary 6.6.8, contrary to the hypothesis.

Lemma 6.6.15. $\forall \Sigma \in \operatorname{Sig}_{\hat{I}}, \forall \psi \in \operatorname{Sen}_{\mathcal{L}}\left(\Sigma_{\mathcal{T}}\right), \exists \psi_{\mathcal{R}} \in \operatorname{Sen}_{\hat{I}}(\Sigma): \forall M \in \operatorname{Mod}_{\hat{I}}(\Sigma):$ $M \vDash_{\hat{I}} \psi_{\mathcal{R}} \Leftrightarrow M_{\mathcal{T}} \vDash_{\mathcal{L}} \psi$

Proof. Let $\mathcal{M}_{\psi}^{\prime}=\left\{M_{1}^{\prime}, \ldots, M_{n}^{\prime}\right\} \subseteq \operatorname{Mod}^{\prime}(\psi)$ such that

$$
\operatorname{Mod}^{\prime}(\psi)=\bigcup_{i \in\{1, \ldots, n\}} \operatorname{Mod}_{\mathcal{L}}\left(\left(\widehat{\varphi}_{M_{i}^{\prime}}\right)_{\mathcal{T}}\right)
$$

according to Lemma 6.6.14, with $\varphi_{M_{1}^{\prime}}, \ldots, \varphi_{M_{n}^{\prime}}$ first-order sentences. Let $\psi_{\mathcal{R}}=$ $\left[\varphi_{M_{1}^{\prime}} \vee \ldots \vee \varphi_{M_{n}^{\prime}}\right]_{\Xi_{I}}$. Then

$$
\begin{aligned}
& M \vDash_{\hat{I}} \psi_{\mathcal{R}} \\
& \quad \Leftrightarrow M \vDash_{\hat{I}} \widehat{\varphi}_{M_{i}^{\prime}} \text { for some } i \in\{1, \ldots, n\}
\end{aligned}
$$


$\Leftrightarrow M_{\mathcal{T}} \vDash_{\mathcal{L}} \widehat{\varphi}_{M_{i}^{\prime} \mathcal{T}}$ for some $i \in\{1, \ldots, n\}$, by the satisfaction invariant, $\Leftrightarrow M_{\mathcal{T}} \vDash_{\mathcal{L}} \psi$, according to $(*)$ above, and since all models in $M_{\mathcal{T}}$ are $\mathcal{L}$-equivalent, according to Lemma 6.6.6.

LEMma 6.6.16. With the notation of Lemma 6.6.15, $\forall M^{\prime} \in \operatorname{Mod}_{\mathcal{L}}\left(\Sigma_{\mathcal{T}}\right): M^{\prime} \vDash_{\mathcal{L}}$ $\psi \Leftrightarrow M_{\mathcal{R}}^{\prime} \vDash_{\hat{I}} \psi_{\mathcal{R}}$.

Proof.

$$
\begin{aligned}
M^{\prime} \vDash_{\mathcal{L}} \psi & \Leftrightarrow\left(M_{\mathcal{R}}^{\prime}\right)_{\mathcal{T}} \vDash_{\mathcal{L}} \psi(\text { by Lemmas } 6.6 .4,6.6 .6 \text { and } 6.6 .7) \\
& \Leftrightarrow M_{\mathcal{R}}^{\prime} \vDash_{\hat{I}} \psi_{\mathcal{R}} \text { (by Lemma 6.6.15). }
\end{aligned}
$$

Lemma 6.6.17. For every $\Sigma \in \operatorname{Sig}_{\hat{I}}$, for every mapping $-\mathcal{R}: \operatorname{Sen}_{\mathcal{L}}\left(\Sigma_{\mathcal{T}}\right) \rightarrow$ $\operatorname{Sen}_{\hat{I}}(\Sigma): \psi \mapsto \psi_{\mathcal{R}}$ (according to Lemma 6.6.15), the corresponding mapping $-\mathcal{R}: \operatorname{Pre}_{\mathcal{L}}\left(\Sigma_{\mathcal{T}}\right) \rightarrow \operatorname{Pre}_{\hat{I}}(\Sigma): E^{\prime} \mapsto E_{\mathcal{R}}^{\prime}$ defined by $E_{\mathcal{R}}^{\prime}=\left\{\psi_{\mathcal{R}} \mid \psi \in E^{\prime}\right\}$ satisfies: $\forall E^{\prime} \in \operatorname{Pre}_{\mathcal{L}}\left(\Sigma_{\mathcal{T}}\right): \forall M^{\prime} \in \operatorname{Mod}_{\mathcal{L}}\left(\Sigma_{\mathcal{T}}\right): M^{\prime} \vDash_{\mathcal{L}} E^{\prime} \Leftrightarrow M_{\mathcal{R}}^{\prime} \vDash_{\hat{I}} E_{\mathcal{R}}^{\prime}$.

P r o of. Immediate consequence of Lemma 6.6.16 and of the elementwise construction of $E_{\mathcal{R}}^{\prime}$.

Lemma 6.6.18. $\forall \tau^{\prime}: \Sigma_{1}^{\prime} \rightarrow \Sigma_{2}^{\prime} \in \operatorname{Sig}_{\mathcal{L}}, \forall E^{\prime} \in \operatorname{Pre}_{\mathcal{L}}\left(\Sigma_{2}^{\prime}\right): \tau_{\mathcal{R}}^{\prime} E_{\mathcal{R}}^{\prime}=\left(\tau^{\prime} E^{\prime}\right)_{\mathcal{R}}$, with $E_{\mathcal{R}}^{\prime}$ as in Lemma 6.6.17.

Proof. Because of the elementwise construction of $E_{\mathcal{R}}^{\prime}$, it is sufficient to show that $\tau_{\mathcal{R}}^{\prime} \psi_{\mathcal{R}}=\left(\tau^{\prime} \psi\right)_{\mathcal{R}}$ for every $\psi \in \operatorname{Sen}_{\mathcal{L}}\left(\Sigma_{2}^{\prime}\right)$, with $\psi_{\mathcal{R}}$ as in Lemma 6.6.15. Since $\hat{I}$ is abstract, by Definition 5.1 it is sufficient to show that $\operatorname{Mod}_{\hat{I}}\left(\tau_{\mathcal{R}}^{\prime} \psi_{\mathcal{R}}\right)=$ $\operatorname{Mod}_{\hat{I}}\left(\left(\tau^{\prime} \psi\right)_{\mathcal{R}}\right)$. This has the following proof. If $M \in \operatorname{Mod}_{\hat{I}}\left(\Sigma_{2 \mathcal{R}}^{\prime}\right)$, then

$$
\begin{aligned}
M \vDash_{\hat{I}} \tau_{\mathcal{R}}^{\prime} \psi_{\mathcal{R}} & \\
\Leftrightarrow & \left.M \tau_{\mathcal{R}}^{\prime} \vDash_{\hat{I}} \psi_{\mathcal{R}} \text { (by the ps property of } \hat{I}\right) \\
\Leftrightarrow & \left(M \tau_{\mathcal{R}}^{\prime}\right)_{\mathcal{T}} \vDash_{\mathcal{L}} \psi(\text { by Lemma } 6.6 .15) \\
\Leftrightarrow & M_{\mathcal{T}} \tau^{\prime} \vDash_{\mathcal{L}} \psi \\
& (\text { naturality of model transformation in } \mathcal{T} \text { and Definition 6.6.1) } \\
\Leftrightarrow & \left.M_{\mathcal{T}} \vDash_{\mathcal{L}} \tau^{\prime} \psi \text { (by the ps property of } \mathcal{L}\right) \\
\Leftrightarrow & \left.M \vDash_{\hat{I}}\left(\tau^{\prime} \psi\right)_{\mathcal{R}} \text { (by Lemma } 6.6 .15\right) .
\end{aligned}
$$

We proceed to complete the proof of the theorem, as follows.

$\mathcal{R}: \mathcal{L} \rightarrow \hat{I}$ according to Definition 6.6.1, the mapping of models as in Definition 6.6.2(i) and the mapping of presentations as in Lemma 6.6.17, is a pre-institution transformation, since Lemma 6.6.9 ensures naturality of model transformation, Lemma 6.6.18 ensures naturality of presentation transformation, and Lemma 6.6.17 shows validity of the satisfaction invariant. 
That $\mathcal{R}$ is an inverse of $\mathcal{T}$ follows from Proposition 3.5, Lemma 6.6.15, and the construction of the mapping of presentations as in Lemma 6.6.17.

Full adequacy of $\mathcal{R}$ follows from Proposition 3.7 and Lemma 6.6.5.

Expansion-adequacy of $\mathcal{R}$ is shown as follows: $\forall \tau^{\prime}: \Sigma_{1}^{\prime} \rightarrow \Sigma_{2}^{\prime} \in \operatorname{Sig}_{\mathcal{L}}, \forall M_{2} \in$ $\operatorname{Mod}_{\hat{I}}\left(\Sigma_{2 \mathcal{R}}^{\prime}\right), \forall M_{1}^{\prime} \in \operatorname{Mod}_{\mathcal{L}}\left(\Sigma_{1}^{\prime}\right)$ :

$$
\begin{aligned}
& M_{2} \tau_{\mathcal{R}}^{\prime} \in M_{1 \mathcal{R}}^{\prime} \\
& \quad \Rightarrow M_{1}^{\prime} \in\left(M_{2} \tau_{\mathcal{R}}^{\prime}\right)_{\mathcal{T}}(\text { by Definition 6.6.2(i)) } \\
& \quad \Rightarrow M_{1}^{\prime} \in M_{2 \mathcal{T}} \tau^{\prime}
\end{aligned}
$$

(naturality of model transformation in $\mathcal{T}$ and Definition 6.6.1)

$$
\begin{aligned}
& \Rightarrow \exists M_{2}^{\prime} \in \operatorname{Mod}_{\mathcal{L}}\left(\Sigma_{2}^{\prime}\right): M_{1}^{\prime}=M_{2}^{\prime} \tau^{\prime} \wedge M_{2}^{\prime} \in M_{2 \mathcal{T}} \\
& \Rightarrow \exists M_{2}^{\prime} \in \operatorname{Mod}_{\mathcal{L}}\left(\Sigma_{2}^{\prime}\right): M_{1}^{\prime}=M_{2}^{\prime} \tau^{\prime} \wedge M_{2} \in M_{2 \mathcal{R}}^{\prime} \text { (by Definition 6.6.2(i)). }
\end{aligned}
$$

Finitarity of $\mathcal{R}$ is an immediate consequence of Lemma 6.6.15 and of the construction of the mapping of presentations as in Lemma 6.6.7.

As we mentioned above, Theorem 6.6 does not generalize the recalled lemma from [6] because it only applies to abstract pre-institutions. A proper generalization of the recalled lemma does exist, however, and is as follows.

In the first place, the hypothesis that $\mathcal{L}$ is abstract plays no rôle in the proof of Theorem 6.6, thus it can be removed.

In the second place, we note that the only place where the abstractness of $\hat{I}$ is made use of in the proof of Theorem 6.6 is Lemma 6.6.18, which shows the naturality of presentation transformation by $\mathcal{R}$.

Now, consider the variant of Theorem 6.6 that is obtained by replacing $\hat{I}$ with $\mathcal{I}_{\mathcal{L}_{I}}$, the first-order pre-institution with only renamings as signature morphismsaccording to Example 2.9, and by relaxing finitarity of $\mathcal{R}$ to quasi-finitarity. This variant of Theorem 6.6 holds as well, as we are going to argue by a simple proof adaptation, and it is easy to see that it properly generalizes the recalled lemma from [6].

The required proof adaptation is as follows.

Starting from Lemma 6.6 .15 , the abstract first-order sentence $\psi_{\mathcal{R}}$ is now to be seen as a first-order presentation. Then the definition of $E_{\mathcal{R}}^{\prime}$ in Lemma 6.6.17 becomes $E_{\mathcal{R}}^{\prime}=\bigcup_{\psi \in E^{\prime}} \psi_{\mathcal{R}}$, which clearly makes $\mathcal{R}$ meet quasi-finitarity. Finally, consider Lemma 6.6.18. $\psi_{\mathcal{R}}$ is now a first-order presentation; more precisely, it is the elementary equivalence class of a first-order sentence. So is $\tau_{\mathcal{R}}^{\prime} \psi_{\mathcal{R}}$, since $\tau_{\mathcal{R}}^{\prime}$ is a first-order signature isomorphism. And obviously, so is $\left(\tau^{\prime} \psi\right)_{\mathcal{R}}$ as well. Since the presentations $\tau_{\mathcal{R}}^{\prime} \psi_{\mathcal{R}}$ and $\left(\tau^{\prime} \psi\right)_{\mathcal{R}}$ have exactly the same models, by a proof analogous to that of Lemma 6.6.18, and since both presentations are elementary equivalence classes of a first-order sentence, they must consist of the same sentences, i.e. they coincide. The identity $\tau_{\mathcal{R}}^{\prime} E_{\mathcal{R}}^{\prime}=\left(\tau^{\prime} E^{\prime}\right)_{\mathcal{R}}$ then follows from the elementwise construction of $E_{\mathcal{R}}^{\prime}$. 
7. Cardinal pre-institutions and Löwenheim-Skolem properties. To begin with, we note that the properties expressed in the Löwenheim-Skolem theorems (both Downward and Upward, see e.g. [4]) refer to the cardinality of models; moreover, the cardinality of symbol sets (generalized by signatures, in our context) play a rôle in generalizations of these theorems, such as the Löwenheim-SkolemTarski theorem.

In pre-institutions, models as well as signatures are viewed as "points" in the general case, that is, abstraction is made from any internal structure they may have. The treatment of (general forms of) the Löwenheim-Skolem properties thus requires the following concept.

DEFINITION 7.1. A pre-institution with cardinal numbers, $\mathcal{K}=(\mathcal{I}, \#)$, or cardinal pre-institution for short, is a pre-institution $\mathcal{I}$ as in Definition 2.1 together with a function \# that assigns a cardinal number $\# \Sigma$ to each signature $\Sigma$, as well as a cardinal number \# $M$ to each model $M$, also called the power of $\Sigma$ or $M$ respectively, and that meets the following conditions, for all signature morphisms $\tau: \Sigma_{1} \rightarrow \Sigma_{2}$ and $\Sigma_{2}$-models $M:$

1. if $\tau$ is monic then $\# \Sigma_{1} \leq \# \Sigma_{2}$,

2. if $\tau$ is epic then $\# \Sigma_{1} \geq \# \Sigma_{2}$,

3. $\# M \geq \# M \tau$.

In abstract model theory, Löwenheim numbers tell the strength of downward Löwenheim-Skolem theorems. The transfer of their definition to pre-institutions is straightforward (cf. Def. 6.2.1 in [5]).

DeFinition 7.2. Let $\mathcal{K}$ be a cardinal pre-institution and $\kappa$ a cardinal. $l_{\kappa}(\mathcal{K})$ is the least cardinal $\mu$ such that every satisfiable set of sentences of power $\leq \kappa$ has a model of power $\leq \mu$, provided there is such a cardinal; otherwise, $l_{\kappa}(\mathcal{K})=\infty$.

$l(\mathcal{K}) \stackrel{\text { def }}{=} l_{1}(\mathcal{K})$ is called the Löwenheim number of $\mathcal{K}$.

The Löwenheim number of a cardinal pre-institution is thus the least cardinal $\mu$ such that every satisfiable sentence has a model of power at most $\mu$, provided such a cardinal exists. Then, not unlike an abstract logic, a cardinal pre-institution $\mathcal{K}$ has the Löwenheim-Skolem property down to $\lambda$ iff $l(\mathcal{K}) \leq \lambda$.

Hanf numbers are the upward counterpart of Löwenheim numbers.

Definition 7.3. Let $\mathcal{K}$ be a cardinal pre-institution and $\kappa$ a cardinal. $h_{\kappa}(\mathcal{K})$ is the least cardinal $\mu$ such that every set of sentences of power $\leq \kappa$ has models of arbitrarily large cardinality if it has a model of power $\geq \mu$, provided there is such a cardinal; otherwise, $h_{\kappa}(\mathcal{K})=\infty$.

$h(\mathcal{K}) \stackrel{\text { def }}{=} h_{1}(\mathcal{K})$ is called the Hanf number of $\mathcal{K}$.

The Hanf number of a cardinal pre-institution is thus the least cardinal $\mu$ such that every sentence satisfiable by a model of power at least $\mu$ has models of arbitrarily larger power, provided such a cardinal exists. 
A classical result in abstract model theory, viz. the Hanf theorem (1960) (see e.g. Thm. 6.1.4 in [5]), guarantees existence of Hanf numbers for abstract logics under certain "smallness" conditions. The same conditions guarantee existence of Löwenheim numbers as well, by a similar argument (see e.g. Prop. 2.5.2 in [4]). The formulation of analogous conditions for pre-institutions requires the following notion.

Definition 7.4. Let $\mathcal{I}$ be a pre-institution as in Definition 2.1. A renaming in $\mathcal{I}$ is an isomorphism $\varrho: \Sigma_{1} \simeq \Sigma_{2}$ in Sig. Moreover, we say that any two signatures $\Sigma_{1}, \Sigma_{2} \in$ Sig are renaming-equivalent in $\mathcal{I}$, written $\Sigma_{1} \simeq \Sigma_{2}$, iff there exists a renaming $\varrho: \Sigma_{1} \simeq \Sigma_{2}$ in Sig. As a matter of notation, if $\Sigma \in \operatorname{Sig}$, $[\Sigma]$ denotes the renaming-equivalence class that contains $\Sigma$.

For cardinal pre-institutions, the following is hardly surprising.

LEMMA 7.5. If $\varrho: \Sigma_{1} \simeq \Sigma_{2}$ is a renaming in a cardinal pre-institution $\mathcal{K}$ as in Definition 7.1, then $\# \Sigma_{1}=\# \Sigma_{2}$, \# $M_{2}=\# M_{2} \varrho$, and $\# M_{1}=\# M_{1} \varrho^{-1}$, for all $M_{1} \in \operatorname{Mod}\left(\Sigma_{1}\right)$ and $M_{2} \in \operatorname{Mod}\left(\Sigma_{2}\right)$.

P r o of. Immediate consequence of Definitions 7.1 and 7.4.

We are now ready to formulate smallness conditions for pre-institutions, that will permit us to lift the Hanf theorem to our framework.

Definition 7.6 (small pre-institution). A pre-institution $\mathcal{I}$ is small iff it meets the following conditions:

(i) the renaming equivalence of signatures in $\mathcal{I}$ has a small set of equivalence classes,

(ii) for every signature $\Sigma$ in $\mathcal{I}$ the set of $\Sigma$-sentences is small.

Smallness of a cardinal pre-institution $\mathcal{K}=(\mathcal{I}, \#)$ is just smallness of the underlying pre-institution $\mathcal{I}$, thus independent of the size of signatures or of models that is defined by \#.

Finally, the following property is a weaker form of the ps property (see Definition 2.2), since it requires satisfaction invariance only for renamings; this closely reflects the renaming property of general logics, in the sense of [5].

Definition 7.7. Let $\mathcal{I}$ be a pre-institution as in Definition 2.1; $\mathcal{I}$ has the renaming property iff it meets the following requirement:

$\forall \varrho: \Sigma_{1} \simeq \Sigma_{2} \in \operatorname{Sig}: \forall \varphi \in \operatorname{Sen}\left(\Sigma_{1}\right), \forall M \in \operatorname{Mod}\left(\Sigma_{2}\right): \quad M \varrho \vDash \varphi \Leftrightarrow M \vDash \varrho \varphi$.

The lifting of the Hanf theorem to pre-institutions now follows. The set-theoretic axiom of replacement plays a key rôle, as in the proof of the classical Hanf theorem.

Proposition 7.8. Let $\mathcal{K}$ be a cardinal pre-institution as in Definition 7.1. If $\mathcal{K}$ has the renaming property and is small, then both its Löwenheim number and its Hanf number exist. 
P r o of. Let $\mathcal{K}$ be a cardinal pre-institution as in the hypothesis. By the axiom of choice, a small set $H$ of signatures exists such that $\forall \Sigma \in \operatorname{Sig}$ : $[\Sigma] \cap H$ is a singleton. We note that the renaming property of $\mathcal{K}$ has the following consequence: if $\Sigma \simeq \Sigma_{1}$, then a renaming $\varrho: \Sigma \rightarrow \Sigma_{1}$ exists, and by Lemma 7.5 and Definition 7.7 a power-preserving bijection between the models of $\varphi$ and those of $\varrho \varphi$ exists, for every $\varphi \in \operatorname{Sen}(\Sigma)$. Now, for each $\Sigma \in H$ and $\varphi \in \operatorname{Sen}(\Sigma)$, let $\alpha_{\varphi}$ be $\infty$ if $\varphi$ has no model, or otherwise the least cardinal $\mu$ such that $\mu=\# M$ for some model $M$ of $\varphi$. By the axiom of replacement, the set $\left\{\alpha_{\varphi} \mid \varphi \in \operatorname{Sen}(\Sigma), \Sigma \in H\right\}$ is small, thus its least upper bound exists, and this is the Löwenheim number of $\mathcal{K}$ thanks to the aforementioned consequence of the renaming property of $\mathcal{K}$.

The existence of the Hanf number of $\mathcal{K}$ has a similar proof.

For the applicability of our form of the "reduction scheme" to LöwenheimSkolem properties along pre-institution transformations, these need the following property. We say that a transformation on cardinal pre-institutions preserves model power if model power never decreases along the transformation. More precisely:

Definition 7.9. Let $\mathcal{T}: \mathcal{K} \rightarrow \mathcal{K}^{\prime}$ be a transformation on cardinal pre-institutions $\mathcal{K}=(\mathcal{I}, \#), \mathcal{K}^{\prime}=\left(\mathcal{I}^{\prime}, \#^{\prime}\right)$, with $\mathcal{T}: \mathcal{I} \rightarrow \mathcal{I}^{\prime}$ as in Definition 2.3. We say that $\mathcal{T}: \mathcal{K} \rightarrow \mathcal{K}^{\prime}$ preserves model power iff $\forall \Sigma \in \operatorname{Sig}, \forall M \in \operatorname{Mod}(\Sigma), \forall M^{\prime} \in M_{\mathcal{T}}$ : $\# M \leq \#^{\prime} M^{\prime}$.

By way of notation, if $S$ is a small set, let $|S|$ denote its cardinality. We conclude with the following reduction theorem for downward Löwenheim-Skolem properties.

Theorem 7.10. Let $\mathcal{K}, \mathcal{K}^{\prime}$ be cardinal pre-institutions as in Definition 7.9. If there exists an adequate transformation $\mathcal{T}: \mathcal{K} \rightarrow \mathcal{K}^{\prime}$ that preserves model power and meets the following condition for cardinals $\mu, \nu: \forall \Sigma \in \operatorname{Sig}, \forall E \in \operatorname{Pre}(\Sigma)$ : $|E| \leq \mu \Rightarrow\left|\left(E_{\mathcal{T}}-\operatorname{Taut}_{\mathcal{T}}(\Sigma)\right) / \equiv_{\mathcal{I}^{\prime}}\right| \leq \nu$, then $l_{\nu}(\mathcal{K}) \leq l_{\nu}\left(\mathcal{K}^{\prime}\right)$

Proof. Let $\Sigma \in \operatorname{Sig}, E \in \operatorname{Pre}(\Sigma)$, with $|E| \leq \mu, E$ satisfiable. If $M \vDash E$, then $M_{\mathcal{T}} \vDash^{\prime} E_{\mathcal{T}}$ by the satisfaction invariant, and $M_{\mathcal{T}}$ is non-empty. Thus $E_{\mathcal{T}}$ is satisfiable, and $\left|\left(E_{\mathcal{T}}-\operatorname{Taut} \mathcal{T}(\Sigma)\right) / \equiv_{\mathcal{I}^{\prime}}\right| \leq \nu$ by the hypothesis. Now, let $E^{\prime}$ consist of one sentence for each equivalence class in $\left(E_{\mathcal{T}}-\operatorname{Taut}_{\mathcal{T}}(\Sigma)\right) / \equiv_{\mathcal{I}^{\prime}}$, thus $\left|E^{\prime}\right| \leq \nu$. Clearly, $E^{\prime}$ is satisfiable iff so is $E_{\mathcal{T}}-\operatorname{Taut}_{\mathcal{T}}(\Sigma)$; moreover, the adequacy of $\mathcal{T}$ entails that $E_{\mathcal{T}} \vDash \operatorname{Taut}_{\mathcal{T}}(\Sigma)$, by Proposition 3.4. Therefore $E^{\prime}$ is satisfiable because so is $E_{\mathcal{T}}$; the fact that $\left|E^{\prime}\right| \leq \nu$ further entails that $E^{\prime}$ has a model $M^{\prime}$ with $\#^{\prime} M^{\prime} \leq l_{\nu}\left(\mathcal{K}^{\prime}\right)$. Then, by the construction of $E^{\prime}$ and since $E_{\mathcal{T}} \vDash \operatorname{Taut}_{\mathcal{T}}(\Sigma)$, $M^{\prime}$ is a model of $E_{\mathcal{T}}$ as well, hence the adequacy of $\mathcal{T}$ entails the existence of a model $N$ of $E$ such that $M^{\prime} \in N_{\mathcal{T}}$. Since $\mathcal{T}$ preserves model power, we infer $\# N \leq \#^{\prime} M^{\prime} \leq l_{\nu}\left(\mathcal{K}^{\prime}\right)$. Thus every satisfiable $E$ of power at most $\mu$ has a model of power at most $l_{\nu}\left(\mathcal{K}^{\prime}\right)$, which is what we had to prove. 
8. Related work $\left({ }^{9}\right)$. This work shares with $[2]$ the motivation for focussing on the morphisms of institution categories more than on their objects. Clearly, a reason for this is the interest that institution-independent specification [19] and general logics [5, 15] naturally find in computer science. It may be source of some surprise, thus, that so far no general agreement has been reached on the most convenient notion of institution morphism. A careful analysis and comparison of several, quite distinct such notions can be found in [2]. To that, we wish to add the following, necessarily quick and preliminary considerations.

In comparison with the notions of institution morphism proposed in $[9,15$, 2 ], our notion is the only one where the three arrows (respectively relating to signatures, sentences, models) are all covariant. Of those three notions, the one which seems closest to ours is that of basic simulation [2], which essentially differs from ours in two respects: 1) it sends sentences to sentences, whereas ours sends presentations to presentations, and 2) model transformation is contravariant, but by a surjective, partial natural transformation. The latter could thus be turned into a covariant, total natural transformation, sending models to sets of models, like in our case. Of course, the model sets ought to be disjoint; this condition, arising from well-definedness of the model transformation as defined for basic simulations, closely corresponds to the sufficient condition for full adequacy given in Lemma 2.6 above - modulo some equivalence of models in the source institution, however.

Although we are more interested in the morphisms than in the objects, the less restrictive definition of the objects in our framework also contributes to widening the applicability of abstract model-theoretic tools in algebraic specification. Most of the specification frameworks studied so far fit the institutions scheme, yet not all of them do. Cases where (at least the eps half of) the satisfaction condition is known to fail include equational type logic with non-standard reduction (as exemplified in Section 5 of [18]) and, most notably, behavioural semantics $[3,16,17]$. These frameworks fit the pre-institutions scheme, as well as the drastically general scheme proposed in [7] — where hardly anything of the abstract model-theoretic approach underlying the theory of institutions can be recognized, however.

9. Future work. We conclude with a list of topics that currently attract our interest, and which seem to deserve further investigation along, and as a test of, the approach presented in this paper.

- Generalization of compactness notions and results to $(\kappa, \lambda)$-compactness [11], which is of interest in the study of infinitary logics.

$\left({ }^{9}\right)$ In this paper, we confine ourselves to the comparison proposed in Section 6 of [18]. In future work of ours we intend to offer a more extensive comparison with related work [1], together with an exploration of connections with other topics in the field of algebraic logic. 
- Investigation of the applicability of the reduction scheme, as generalized here, to other properties such as: Beth definability, Craig interpolation, Robinson property, Karp property, freeness, initiality, etc.

- Lindström theorems.

- Expressiveness applications.

\section{References}

[1] H. Andréka, I. Németi and I. Sain, Abstract model theoretic approach to algebraic logic, manuscript, Mathematical Institute, Budapest, 1984.

[2] E. Astesiano and M. Cerioli, Commuting between institutions via simulation, Univ. of Genova, Formal Methods Group, Technical Report no. 2, 1990.

[3] G. Bernot and M. Bidoit, Proving the correctness of algebraically specified software: Modularity and Observability issues, in: M. Nivat, C. M. I. Rattray, T. Rus and G. Scollo (eds.), AMAST '91, Algebraic Methodology and Software Technology, Workshops in Computing, Springer, London 1992, 216-239.

[4] C. C. Chang and H. J. Keisler, Model Theory, third ed., North-Holland, Amsterdam 1990.

[5] H.-D. Ebbinghaus, Extended logics: the general framework, in: J. Barwise and S. Feferman (eds.), Model-Theoretic Logics, Springer, Berlin 1985, 25-76.

[6] H.-D. Ebbinghaus, J. Flum and W. Thomas, Mathematical Logic, Springer, New York 1984.

[7] H. Ehrig, M. Baldamus, F. Cornelius and F. Orejas, Theory of algebraic module specification including behavioural semantics and constraints, in: M. Nivat, C. M. I. Rattray, T. Rus and G. Scollo (eds.), AMAST '91, Algebraic Methodology and Software Technology, Workshops in Computing, Springer, London 1992, 145-172.

[8] A. J. M. van Gasteren, On the Shape of Mathematical Arguments, Lecture Notes in Comput. Sci. 445, Springer, Berlin 1990.

[9] J. A. Goguen and R. Burstall, Introducing institutions, in: E. Clarke and D. Kozen (eds.), Logics of Programs, Lecture Notes in Comput. Sci. 164, Springer, Berlin 1984, 221-256.

[10] S. MacLane, Categories for the Working Mathematician, Graduate Texts in Math. 5, Springer, New York 1971.

[11] J.-A. Makowski, Compactness, embeddings and definability, in: J. Barwise and S. Feferman (eds.), Model-Theoretic Logics, Springer, Berlin 1985, 645-716.

[12] V. Manca and A. Salibra, On the power of equational logic: applications and extensions, in: H. Andréka, J. D. Monk and I. Németi (eds.), Algebraic Logic, Colloq. Math. Soc. János Bolyai 54, North-Holland, Amsterdam 1991, 393-412.

[13] V. Manca, A. Salibra and G. Scollo, Equational type logic, Theoret. Comput. Sci. 77 (1990), 131-159.

[14] - , - - - On the expressiveness of equational type logic, in: C. M. I. Rattray and R. G. Clark (eds.), The Unified Computation Laboratory: Modelling, Specifications and Tools, Oxford Univ. Press, Oxford 1992, 85-100.

[15] J. Meseguer, General logics, in: H.-D. Ebbinghaus et al. (eds.), Logic Colloquium '87, North-Holland, Amsterdam 1989, 275-329.

[16] M. P. Nivela and F. Orejas, Initial behaviour semantics for algebraic specifications, in: D. T. Sannella and A. Tarlecki (eds.), Recent Trends in Data Type Specification, Lecture Notes in Comput. Sci. 332, Springer, Berlin 1988, 184-207. 
[17] F. Orejas, M. P. Nivela and H. Ehrig, Semantical constructions for categories of behavioural specifications, in: H. Ehrig, H. Herrlich, H.-J. Kreowski and G. Preuß (eds.), Categorical Methods in Computer Science - with Aspects from Topology, Lecture Notes in Comput. Sci. 393, Springer, Berlin 1989, 220-245.

[18] A. Salibra and G. Scollo, A soft stairway to institutions, in: M. Bidoit and C. Choppy (eds.), Recent Trends in Data Type Specification, Lecture Notes in Comput. Sci. 655, Springer, Berlin 1993, 310-329.

[19] D. T. Sannella and A. Tarlecki, Specifications in an arbitrary institution, Inform. and Comput. 76 (1988), 165-210. 